Números irracionais: $\pi$ e e

Silvana de Lourdes Gálio Spolaor 

SERVIÇO DE PÓS-GRADUAÇÃO DO ICMC-USP

Data de Depósito:

Assinatura:

\title{
Números irracionais: $\pi$ e e
}

\section{Silvana de Lourdes Gálio Spolaor}

\section{Orientador: Prof. Dr. Paulo Leandro Dattori da Silva}

\begin{abstract}
Dissertação apresentada ao Instituto de Ciências Matemáticas e de Computação - ICMC-USP, como parte dos requisitos para obtenção do título de Mestre em Ciências - Programa de Mestrado Profissional em Matemática . VERSÃO REVISADA.
\end{abstract}


Ficha catalográfica elaborada pela Biblioteca Prof. Achille Bassi e Seção Técnica de Informática, ICMC/USP, com os dados fornecidos pelo(a) autor(a)

\begin{tabular}{|c|c|}
\hline \multirow[t]{3}{*}{ S762n } & $\begin{array}{l}\text { Spolaor, Silvana de Lourdes Gálio Spolaor } \\
\quad \text { Números irracionais: Ipi e e. / Silvana de } \\
\text { Lourdes Gálio Spolaor Spolaor; orientador Paulo } \\
\text { Leandro Dattori da Silva Dattori da Silva. -- São } \\
\text { Carlos, 2013. } \\
\quad 41 \mathrm{p} \text {. }\end{array}$ \\
\hline & $\begin{array}{l}\text { Tese (Doutorado - Programa de Pós-Graduação em } \\
\text { Mestrado Profissional em Matemática em Rede } \\
\text { Nacional) -- Instituto de Ciências Matemáticas e de } \\
\text { Computação, Universidade de São Paulo, } 2013 \text {. }\end{array}$ \\
\hline & $\begin{array}{l}\text { 1. números reais. 2. números irracionais. } 3 . \\
\text { Número de Euler. 4. número \pi. I. Dattori da } \\
\text { Silva, Paulo Leandro Dattori da Silva, orient. II. } \\
\text { Título. }\end{array}$ \\
\hline
\end{tabular}


Ao meu esposo, Éder Marcos, $e$ aos meus filhos, Bruno e Vítor. 

"Deus criou os números naturais e o resto é obra da humanidade." - Leopold Kronecker(1893) 



\section{Agradecimentos}

A Deus, por todos os dias da minha vida e por estar comigo nos momentos mais difíceis.

À minha família pelo apoio, em especial ao meu esposo, pelo incentivo e principalmente pelos conselhos e palavras motivadoras.

À coordenadora do PROFMAT, Prof ${ }^{\mathrm{a}} \mathrm{Dr}^{\mathrm{a}}$ Ires Dias, pela paciência, carinho e ensinamentos, contribuindo de forma ímpar para meu crescimento profissional e pessoal.

Ao meu orientador Prof. Dr. Paulo Dattori, pelo apoio, dedicação, confiança e pelos importantes ensinamentos que tornaram possível a conclusão desta dissertação.

Aos professores do ICMC, especialmente aos professores Ladeira, Hermano, Miguel e Zani pelos conhecimentos disponibilizados durante as aulas, contribuindo de forma significativa para meu aprimoramento profissional.

À Coordenação de Aperfeiçoamento de Pessoal de Nível Superior (CAPES) pelo apoio financeiro. 

Nesta dissertação são apresentadas algumas propriedades de números reais.

Descrevemos de maneira breve os conjuntos numéricos $\mathbb{N}, \mathbb{Z}, \mathbb{Q}$ e $\mathbb{R}$ e apresentamos demonstrações detalhadas da irracionalidade dos números $\pi$ e $e$.

Também, apresentamos um texto sobre o número e, menos técnico e mais intuitivo, na tentativa de auxiliar o professor no preparo de aulas sobre o número e para alunos do Ensino Médio, bem como, alunos de cursos de Licenciatura em Matemática. 

In this thesis we present some properties of real numbers.

We describe briefly the numerical sets $\mathbb{N}, \mathbb{Z}, \mathbb{Q}$ and $\mathbb{R}$, and we present detailed proofs of irrationality of numbers $\pi$ and $e$.

We also present a text about the number $e$ less technical and more intuitive in an attempt to assist the teacher in preparing lessons about number $e$ for High School students as well as for Teaching degree in Mathematics students. 



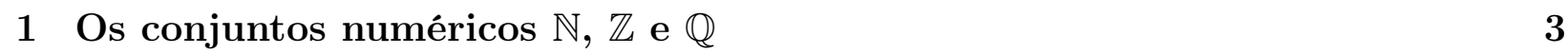

2 A não completude de $\mathbb{Q}$ e o conjunto dos números reais $\quad 7$

3 O número $\pi \quad 13$

3.1 A irracionalidade de $\pi \ldots \ldots \ldots \ldots \ldots$

4 O número e $r$

4.1 A irracionalidade do número e . . . . . . . . . . . . . . 24

$5 \quad$ O número e: possibilidade para a sala de aula $\quad 27$

5.1 Surgimento do número e . . . . . . . . . . . . . . . . . . . . 27

5.2 A irracionalidade de $e \ldots \ldots \ldots \ldots$

6 Considerações finais $\quad 39$

$\begin{array}{ll}\text { Referências Bibliográficas } & 39\end{array}$ 



\section{Introdução}

Um tema recorrente aos Professores de Matemática tanto do ensino básico quanto superior é como ensinar os conjuntos numéricos $\mathbb{N}, \mathbb{Z}, \mathbb{Q}$ e $\mathbb{R}$.

Enquanto no ensino superior tais conjuntos são estudados sob o ponto de vista da álgebra e da análise, no ensino básico nos contentamos apenas em apresentá-los como conjuntos numéricos e exploramos propriedades da adição e multiplicação apenas como "operações básicas" destes conjuntos.

O que nos chamou a atenção é que alguns livros didáticos do ensino básico, como por exemplo [2], [3], [4], [8] e [15], descrevem o conjunto dos números reais como sendo $\mathbb{R}=\mathbb{Q} \cup(\mathbb{R} \backslash \mathbb{Q}) ;$ porém, quem é $\mathbb{R} \backslash \mathbb{Q}$ ? Como caracterizar os elementos de $\mathbb{R} \backslash \mathbb{Q}$ ? Note que os referidos livros didáticos descrevem os conjuntos $\mathbb{N}, \mathbb{Z}$ e $\mathbb{Q}$.

Não é difícil verificar que $\mathbb{R} \backslash \mathbb{Q} \neq \emptyset$. Por exemplo, fazendo uso do teorema de Pitágoras podemos facilmente verificar que deve existir um número $x$ tal que $x^{2}=2$; tal número é denotado por $\sqrt{2}$. Afirmamos que $\sqrt{2}$ é um número irracional. Alguns livros didáticos do ensino básico apresentam uma demonstração de tal fato (ver, por exemplo, [3], [4] e [15]). Esta demonstração é elementar e pode ser apresentada e discutida com alunos do final do ensino fundamental e alunos do ensino médio, pois trata-se de uma prova simples e os argumentos utilizados exigem apenas propriedades de números pares e ímpares.

Usando propriedades de números primos é possível verificar que se $p$ é primo então $\sqrt{p}$ é irracional. Esta demonstração é um pouco mais elaborada, porém, razoavelmente simples de ser feita. De fato, a demonstração utiliza (apenas) argumentos de divisibilidade. Logo, temos aqui infinitos (porém, enumeráveis) exemplos de números irracionais.

Propriedades importantes do conjunto dos números irracionais e, consequentemente, do conjunto dos números reais não são estudadas no ensino básico; por exemplo, $\mathbb{R} \backslash \mathbb{Q}$ não é enumerável.

Nem todo número irracional é da forma $\sqrt{\mathrm{p}}$; por exemplo, o número $\pi$ (pois, $\pi$ não é algébrico - ver [6]). O número $\pi$ aparece nos livros didáticos do ensino básico como a razão entre o perímetro de uma circunferência e o seu diâmetro. Porém, no ensino básico apenas ouvimos falar que $\pi$ é irracional! Então, o que dizer do número $\pi$ ? É de fato um irracional? 
Embora o número $\pi$ seja estudado (pelo menos) desde a Grécia antiga, apenas no século XVIII foi provada sua irracionalidade.

Em 1761, Johann Heinrich Lambert provou que $\pi$ é um número irracional. De fato, Lambert provou que se $0 \neq x \in \mathbb{Q}$ então $\tan (x)$ não é racional; note que $\tan \left(\frac{\pi}{4}\right)=1 \mathrm{e}$, portanto, $\frac{\pi}{4}$ não é racional.

Nesta dissertação pretendemos, além de discutir a "natureza" dos números irracionais (definição, existência e propriedades), apresentar uma demonstração da irracionalidade de $\pi$ (ver capítulo 3). Optamos pela demonstração apresentada por Ivan Niven em 1947 no artigo intitulado "A simple proof that $\pi$ is irrational" e publicado no Bulletin of the American Mathematical Society (ver [12]). Aqui apresentaremos, com detalhes, a demonstração da irracionalidade de $\pi$ conforme encontrado em [6].

Não é nossa pretensão que o aluno de ensino médio ou fundamental conheça as argumentações utilizadas para provar a irracionalidade de $\pi$, pois esta exige conhecimentos prévios de Cálculo Diferencial e Integral, conteúdo não abordado no ensino fundamental e médio, em geral.

Outro exemplo de número irracional que aparece nos livros de ensino médio é o número e, conhecido como número de Euler. Até onde pudemos verificar e é apresentado apenas como um irracional cujo valor aproximado é 2,7182818 … Leonhard Euler (1707-1783), matemático suíço, foi um dos primeiros a estudar as propriedades deste número. Porém, como mencionado por Maor em [11], as origens de e são anteriores a Euler e aparentemente surgiram em problemas de juros. Aqui apresentaremos uma demonstração detalhada da irracionalidade de e (ver capítulo 4) baseada naquela apresentada em [11].

Em [7] a irracionalidade de e também é provada, porém com uma argumentação um pouco diferente; de fato, em [7] a irracionalidade de e é vista como uma aplicação da fórmula de Taylor.

Ao contrário do número $\pi$, acreditamos que seja possível discutir a demonstração da irracionalidade de e com os alunos do ensino médio. Podemos definir e, e verificar sua irracionalidade, utilizando apenas propriedades de sequências e séries. No capítulo 5, apresentamos um texto com uma abordagem diferente daquela que utilizamos no capítulo 4; optamos por utilizar uma linguagem menos técnica e mais intuitiva na tentativa de tornar o texto mais agradável e acessivel. Acreditamos que o texto que será apresentado no capítulo 5 possa ser utilizado para formar um conjunto de aulas para o Ensino Médio, bem como, para alunos de cursos de Licenciatura em Matemática.

Ao abordarmos, neste trabalho, os conjuntos numéricos, não pretendemos fazer a definição axiomática dos mesmos, pois este não é o foco desta dissertação. Procuramos, de maneira breve, definir o conjunto dos números racionais e apresentamos algumas propriedades deste conjunto. Em especial, verificamos que o conjunto dos números racionais com as operações de soma e multiplicação usuais é um corpo ordenado, mas não é completo.

Por fim, adotamos (como em [10]) o axioma fundamental da Análise Matemática, que indica a existência de um corpo ordenado completo, que denotamos por $\mathbb{R}$. Enunciamos o fato de $\mathbb{R}$ não ser enumerável e, consequentemente, o conjunto dos números irracionais não é enumerável. 


$\frac{2 m}{1}$

\section{Os conjuntos numéricos $\mathbb{N}, \mathbb{Z}$ e $\mathbb{Q}$}

O objetivo deste capítulo é apresentar uma breve descrição dos conjuntos numéricos $\mathbb{N}, \mathbb{Z}$ e $\mathbb{Q}$.

Podemos estabelecer, axiomaticamente, que o conjunto dos números naturais é o conjunto $\mathbb{N}$, que satisfaz os conhecidos axiomas de Peano:

(A1) Todo número natural tem um único sucessor;

(A2) Números naturais diferentes tem sucessores diferentes;

(A3) Existe um único número natural, chamado um e representado pelo símbolo 1, que não é sucessor de nenhum outro;

(A4) Seja $X$ um conjunto de números naturais (isto é, $X \subset \mathbb{N}$ ). Se $1 \in X$ e se, além disso, o sucessor de todo elemento de $X$ ainda pertence a $X$, então $X=\mathbb{N}$.

Utilizando o conjunto dos números naturais podemos definir o conjunto dos números inteiros, denotado por $\mathbb{Z}$, o qual a menos de um isomorfismo de anéis ordenados é o único domínio bem ordenado (ver teorema 2.3 da seção 3.10 de [5]).

Finalmente, utilizando o conjunto dos inteiros podemos definir o conjunto dos números racionais, denotado por $\mathbb{Q}$, como sendo o corpo de frações de $\mathbb{Z}$ (ver capítulo 4 de [5]).

Não é intuito deste texto apresentar as construções algébricas precisas dos conjuntos numéricos $\mathbb{N}, \mathbb{Z}$ e $\mathbb{Q}$, da maneira como foram mencionadas acima. 
De modo "bem simples" vamos descrever os referidos conjuntos da seguinte maneira:

$$
\begin{gathered}
\mathbb{N}=\{1,2,3,4,5,6,7,8,9,10,11,12,13, \ldots\}, \\
\mathbb{Z}=\{\ldots,-3,-2,-1,0,1,2,3, \ldots\}
\end{gathered}
$$

$\mathrm{e}$

$$
\mathbb{Q}=\left\{\frac{p}{q} ; p, q \in \mathbb{Z} \text { e } q \neq 0\right\}
$$

Pelo descrito acima $\mathbb{N} \subset \mathbb{Z} \subset \mathbb{Q}$. Note que se "desenharmos" uma reta podemos representar todos os elementos de $\mathbb{Q}$ nesta reta. Será que todos os pontos de tal reta são representantes de algum elemento de $\mathbb{Q}$ ?

A seguir, vamos apresentar algumas propriedades do conjunto $\mathbb{Q}$.

Propriedade 1. (Igualdade dos números racionais) Sejam $\frac{\mathrm{a}}{\mathrm{b}}$ e $\frac{\mathrm{c}}{\mathrm{d}}$ números racionais. Então, $\frac{\mathrm{a}}{\mathrm{b}}=\frac{\mathrm{c}}{\mathrm{d}}$ se, e somente se, $\mathrm{a} \times \mathrm{d}=\mathrm{b} \times \mathrm{c}$.

Propriedade 2. Todo número racional pode ser representado na forma $\frac{a}{b}$, sendo $b$ um número natural.

Propriedade 3 . Todo número racional $\frac{a}{b}>0$ pode ser escrito na forma $\frac{x}{y}$, sendo $\operatorname{mdc}(x, y)=1$.

Demonstração: De fato, se $d=\operatorname{mdc}(a, b)$, então existem inteiros $x$ e $y$ tais que $a=x \times d$, $b=y \times d$ e $\operatorname{mdc}(x, y)=1$. Logo, $\frac{a}{b}=\frac{x \times d}{y \times d}$, pois $a \times y \times d=x \times d \times b$.

O conjunto dos números racionais com as operações usuais de adição "+" e multiplicação ".", possui uma estrutura algébrica bem forte; mais precisamente, $\mathbb{Q}$ é um corpo. De fato, definindo em $\mathbb{Q}$ soma e multiplicação (usuais) por

- dados dois números racionais $\frac{\mathrm{a}}{\mathrm{b}} \mathrm{e} \frac{\mathrm{c}}{\mathrm{d}}$, definimos a operação "adição" por:

$$
\frac{a}{b}+\frac{c}{d}=\frac{(a \times d)+(b \times c)}{b \times d}
$$

- dados dois números racionais $\frac{\mathrm{a}}{\mathrm{b}} \mathrm{e} \frac{\mathrm{c}}{\mathrm{d}}$, definimos a operação "multiplicação" por:

$$
\frac{a}{b} \cdot \frac{c}{d}=\frac{a \times c}{b \times d}
$$

É fácil ver que:

1. quaisquer que sejam $\frac{a}{b}, \frac{c}{d}, \frac{e}{f} \in \mathbb{Q}$ tem-se $\left(\frac{a}{b}+\frac{c}{d}\right)+\frac{e}{f}=\frac{a}{b}+\left(\frac{c}{d}+\frac{e}{f}\right)$; 
2. quaisquer que sejam $\frac{\mathrm{a}}{\mathrm{b}}, \frac{\mathrm{c}}{\mathrm{d}} \in \mathbb{Q}$ tem-se $\frac{\mathrm{a}}{\mathrm{b}}+\frac{\mathrm{c}}{\mathrm{d}}=\frac{\mathrm{c}}{\mathrm{d}}+\frac{\mathrm{a}}{\mathrm{b}}$;

3. $0 \in \mathbb{Q}$ é tal que $\frac{p}{q}+0=\frac{p}{q}$, seja qual for $\frac{p}{q} \in \mathbb{Q}$;

4. todo elemento $\frac{p}{q} \in \mathbb{Q}$ possui um simétrico $-\frac{p}{q} \in \mathbb{Q}$ tal que $\frac{p}{q}+\left(-\frac{p}{q}\right)=0$;

5. dados quaisquer $\frac{a}{b}, \frac{c}{d}, \frac{e}{f} \in \mathbb{Q}$ tem-se $\left(\frac{a}{b} \cdot \frac{c}{d}\right) \cdot \frac{e}{f}=\frac{a}{b} \cdot\left(\frac{c}{d} \cdot \frac{e}{f}\right)$;

6. sejam quais forem $\frac{a}{b}, \frac{c}{d} \in \mathbb{Q}$ tem-se $\frac{a}{b} \cdot \frac{c}{d}=\frac{c}{d} \cdot \frac{a}{b}$;

7. $1 \in \mathbb{Q}$ é tal que $1 \neq 0$ e $\frac{p}{q} \cdot 1=\frac{p}{q}$, qualquer que seja $\frac{p}{q} \in \mathbb{Q}$;

8. todo $\frac{p}{q} \neq 0$ em $\mathbb{Q}$ possui um inverso $\frac{q}{p}$ tal que $\frac{p}{q} \cdot \frac{q}{p}=1$;

9. dados quaisquer $\frac{a}{b}, \frac{c}{d}, \frac{e}{f} \in \mathbb{Q}$ tem-se $\frac{a}{b} \cdot\left(\frac{c}{d}+\frac{e}{f}\right)=\frac{a}{b} \cdot \frac{c}{d}+\frac{a}{b} \cdot \frac{e}{f}$.

Definição 1.1. Dizemos que um corpo $K$ é ordenado quando existe um subconjunto $\mathrm{K}_{+}$ de K, chamado conjunto dos elementos positivos, satisfazendo as seguintes condições:

$\mathrm{P} 1$ Dados $\mathrm{x}, \mathrm{y} \in \mathrm{K}_{+} \Rightarrow \mathrm{x}+\mathrm{y} \in \mathrm{K}_{+}$e $\mathrm{x} \cdot \mathrm{y} \in \mathrm{K}_{+}$, ou seja, a soma e o produto de elementos positivos são sempre positivos.

P2 Dado $\mathrm{x} \in \mathrm{K}$ apenas uma das seguintes situações ocorre: $\mathrm{x}=0$ ou $\mathrm{x} \in \mathrm{K}_{+}$ou $-x \in K_{+}$.

Exemplo 1.2. $\mathbb{Q}$ é um corpo ordenado. De fato, é fácil ver que o conjunto

$$
\mathbb{Q}_{+}=\left\{\frac{p}{q} \in \mathbb{Q} ; p, q \in \mathbb{N}\right\}
$$

satisfaz as propriedades $\mathbf{P} 1$ e $\mathbf{P 2}$.

Em um corpo ordenado $K$ temos a noção de ordem. Dados $x, y \in K, x \neq y$, denotamos $x<y$ para significar $y-x \in K_{+}$e lê-se $x$ menor do que $y$.

Analogamente, $y>x$ significa $y-x>K_{+}$e lê-se $y$ maior do que $x$.

Proposição 1.3. Dados os números racionais $\frac{p}{q} e \frac{r}{s}$, como $q, s>0$, temos que

$$
\frac{r}{s}>\frac{p}{q} \Leftrightarrow r q>p s
$$


Demonstração: De fato,

$$
\frac{r}{s}>\frac{p}{q} \Leftrightarrow \frac{r q-p s}{q s}=\frac{r}{s}-\frac{p}{q} \in \mathbb{Q}_{+} \Leftrightarrow(r q-p s) q s \in \mathbb{N} \Leftrightarrow r q-p s>0 \Leftrightarrow r q>p s .
$$

Vamos, agora, estudar conjuntos limitados.

Definição 1.4. Seja $A \subset \mathbb{Q}$.

i) Dizemos que $r \in \mathbb{Q}$ é cota superior de $A$ se $x \leq r$, para todo $x \in A$.

ii) Dizemos que $r \in \mathbb{Q}$ é cota inferior de $A$ se $r \leq x$, para todo $x \in A$.

Definição 1.5. Dizemos que um conjunto $A \subset \mathbb{Q}$ é limitado se existe $r \in \mathbb{Q}$ tal que $-r \leq x \leq r$, para todo $x \in A$. Em outras palavras, $A \subset \mathbb{Q}$ é limitado se $A$ admite cota inferior e cota superior. Quando A admite cota superior ou cota inferior, dizemos que $A$ é limitado superiormente ou inferiormente, respectivamente.

Exemplo 1.6. $\mathbb{Z}_{-}=\{\cdots-3,-2,-1\}$. Temos que $-1,-\frac{1}{2}, 0, \frac{1}{4}$ e 1 são exemplos de cotas superiores de $\mathbb{Z}_{-}$. Note que $\mathbb{Z}_{-}$não admite cota inferior.

Exemplo 1.7. $\mathbb{Z}_{+}=\{1,2,3,4,5, \ldots\}$ Temos que $1, \frac{1}{2}, 0,-\frac{1}{2} e-1$ são exemplos de cotas inferiores de $\mathbb{Z}_{+}$. Note que $\mathbb{Z}_{+}$não admite cota superior.

Exemplo 1.8. $A=\{1,2,3\}$ é limitado.

Definição 1.9. Seja $A \subset \mathbb{Q}$ limitado inferiormente. Dizemos que $\alpha$ é o infimo de $A$ se $\alpha$ é a maior das cotas inferiores de A.

Notação: $\alpha=\inf A$.

Exemplo 1.10. 1 é infimo de $\mathbb{Z}_{+}$.

Definição 1.11. Seja $\mathrm{B} \subset \mathbb{Q}$ limitado superiormente. Dizemos que $\beta$ é o supremo de $\mathrm{B}$ se $\beta$ é a menor das cotas superiores de B.

Notação: $\beta=\sup B$.

Exemplo 1.12. -1 é supremo de $\mathbb{Z}_{-}$.

Os exemplos acima sugerem a seguinte pergunta natural: será que sempre podemos obter o supremo e o ínfimo de conjuntos limitados de $\mathbb{Q}$ ?

Exemplo 1.13. Se $\emptyset \neq X \subset \mathbb{Z}(\subset \mathbb{Q})$ é limitado então existem $\mathrm{a}, \mathrm{b} \in \mathrm{X}$ tais que $\mathrm{a}=\inf \mathrm{X}$ $e \mathrm{~b}=\sup X$.

De fato, como $\emptyset \neq X \subset \mathbb{Z}$ é limitado, $X$ possui no máximo um número finito de elementos. Logo, X possui um maior elemento e um menor elemento. Denotemos $\mathrm{b}=$ maior elemento de $\mathrm{X}$ e $\mathrm{a}=$ menor elemento de $\mathrm{X}$. Então, $\mathrm{b}=\sup \mathrm{X}$ e $\mathrm{a}=\inf \mathrm{X}$.

Como consequência do exemplo acima, temos:

1. Para todo $\emptyset \neq X \subset \mathbb{Z}$ limitado superiormente existe $\sup X$.

2. Para todo $\emptyset \neq X \subset \mathbb{Z}$ limitado inferiormente existe inf $X$.

No próximo capítulo veremos que $\mathbb{Q}$ não satisfaz as propriedades 1 e 2 acima. 


$\frac{2 m}{2}$

\section{A não completude de $\mathbb{Q}$ e o conjunto dos números reais}

O objetivo deste capítulo é mostrar que sob certo aspecto, o conjunto dos números racionais, denotado por $\mathbb{Q}$, possui "buracos" e, então, mostrar a necessidade de construir um conjunto que contenha $\mathbb{Q}$ e que preencha esses "buracos". O primeiro passo é mostrar que não existe $0<\frac{p}{q} \in \mathbb{Q}, \operatorname{com} \operatorname{mdc}(p, q)=1$ tal que $\left(\frac{p}{q}\right)^{2}=2$; isto será consequência das duas propriedades de números naturais abaixo:

P1. Se $n \in \mathbb{N}$ é par então $n^{2}$ é par.

De fato, se $n \in \mathbb{N}$ é par então existe $m \in \mathbb{N}$ tal que $n=2 m$. Daí, $n^{2}=4 m^{2}=2.2 m^{2}$, com $2 m^{2} \in \mathbb{N}$. Portanto, $n^{2}$ é par.

P2. Se $n \in \mathbb{N}$ é ímpar então $n^{2}$ é ímpar.

De fato, se $n \in \mathbb{N}$ é ímpar então existe $m \in \mathbb{N}$ tal que $n=2 m+1$. Daí, $n^{2}=(2 m+1)^{2}=$ $4 m^{2}+4 m+1=2\left(2 m^{2}+2 m\right)+1, \operatorname{com}\left(2 m^{2}+2 m\right) \in \mathbb{N}$. Portanto, $n$ é ímpar.

Proposição 2.1. Não existe $\frac{p}{q} \in \mathbb{Q}_{+}$tal que $\left(\frac{p}{q}\right)^{2}=2$. 
Demonstração: Suponha, por absurdo, que existam $p, q \in \mathbb{N}$, com $\operatorname{mdc}(p, q)=1$, tais que $\left(\frac{p}{q}\right)^{2}=2$. Daí, $2=\frac{p^{2}}{q^{2}} \Rightarrow p^{2}=2 q^{2} \Rightarrow p^{2}$ é par. Então, pelas propriedades $\mathbf{P} 1$ e $\mathbf{P} 2$, $p$ é par. Consequentemente, existe $m \in \mathbb{N}$ tal que $p=2 m$. Logo, $(2 m)^{2}=2 q^{2} \Rightarrow 4 m^{2}=$ $2 \mathrm{q}^{2} \Rightarrow \mathrm{q}^{2}=2 \mathrm{~m}^{2} \Rightarrow \mathrm{q}^{2}$ é par. Então, pelas propriedades $\mathrm{P} 1$ e P2, q é par.

Como q é par e $p$ é par temos que $1=\operatorname{mdc}(p, q) \geq 2$. Absurdo.

Sejam

$$
X=\left\{x \in \mathbb{Q} ; x \geq 0 \text { e } x^{2}<2\right\}
$$

e

$$
\mathrm{Y}=\left\{\mathrm{y} \in \mathbb{Q} ; \mathrm{y}>0 \text { e } \mathrm{y}^{2}>2\right\} .
$$

Note que $Y \subset(0,+\infty)$. Além disso, se $x>2$ então $x^{2}>4$; consequentemente $X \subset[0,2]$.

Vamos mostrar que não existem $r_{1}, r_{2} \in \mathbb{Q}$ tais que $r_{1}=\sup X$ e $r_{2}=\inf Y$. Antes provaremos três lemas auxiliares.

Lema 2.2. $X$ não possui um maior elemento. Em outras palavras, não existe $x_{0} \in X$ tal que $x_{0} \geq x, \forall x \in X$.

Demonstração: Para demonstrar a afirmação devemos, para cada $\chi \in X$, encontrar $\chi^{\prime} \in$ $X, \operatorname{com} x<x^{\prime}$. Agora, $x^{\prime}>x$ significa que $x^{\prime}=x+r$, para algum $0<r \in \mathbb{Q}_{+}$.

Logo, para cada $x \in X$ basta mostrar que existe $0<r \in \mathbb{Q}_{+}$tal que $x+r \in X$.

Note que $x=1 \in X$, pois $1>0$ e $1^{2}=1<2$; consequentemente, se $X$ tiver um maior elemento, digamos $x_{0}$, então $x_{0} \geq 1$.

Logo, basta mostrar que para todo $x \in X$, com $x \geq 1$, existe $0<r \in \mathbb{Q}_{+}$tal que $x+r \in X$. Note que para $x \in X$, com $x \geq 1$, um tal $r \in \mathbb{Q}_{+}$deve ser menor que 1 , pois caso contrário

$$
(x+r)^{2}=x^{2}+2 x r+r^{2}>x^{2}+r^{2} \geq 1+1=2 .
$$

Logo, para cada $x \in X$, com $x \geq 1$, devemos encontrar $r \in \mathbb{Q}_{+}$, com $0<r<1$, tal que $(x+r)^{2}<2$

Agora, para $0<r<1$ temos

$$
(x+r)^{2}=x^{2}+2 x r+r^{2}=x^{2}+(2 x+r) r<x^{2}+(2 x+1) r .
$$

Daí, para cada $x \in X$ basta encontrar $0<r<1$ tal que $x^{2}+(2 x+1) r<2$; equivalentemente, $0<r<\frac{2-x^{2}}{2 x+1}$

Logo, para cada $x \in X$, tome $r \in \mathbb{Q}$ com $0<r<\min \left\{1, \frac{2-x^{2}}{2 x+1}\right\}$.

Daí, $r^{2}<r$ e $r(2 x+1)<2-x^{2}$; consequentemente,

$$
(x+r)^{2}=x^{2}+2 x r+r^{2}<x^{2}+2 x r+r=x^{2}+r(2 x+1)<x^{2}+2-x^{2}=2
$$

e, portanto, $x+r \in X$.

Lema 2.3. $Y$ não possui um menor elemento. Em outras palavras, não existe $y_{0} \in Y$ tal que $\mathrm{y}_{0} \leq \mathrm{y}, \forall \mathrm{y} \in \mathrm{Y}$. 
Demonstração: Para demonstrar a afirmação devemos, para cada $y \in Y$, encontrar $\mathrm{y}^{\prime} \in \mathrm{Y}, \operatorname{com} \mathrm{y}^{\prime}<\mathrm{y}$.

Note que $y^{\prime}<y$ significa que $y^{\prime}=y-r$, para algum $0<r \in \mathbb{Q}$.

Logo, para cada $y \in Y$ devemos mostrar que existe $0<r \in \mathbb{Q}$ tal que $y-r \in Y$ (isto é, $\left.(y-r)^{2}>2\right)$. Note que para $0<r$ tem-se

$$
(y-r)^{2}=y^{2}-2 y r+r^{2}>y^{2}-2 y r
$$

portanto, basta encontrar $0<r$ tal que

$$
y^{2}-2 y r>2
$$

equivalentemente,

$$
r<\frac{y^{2}-2}{2 y}
$$

Logo, para cada $y \in Y$ tome $r \in \mathbb{Q}$, com $0<r<\frac{y^{2}-2}{2 y}$. Daí, $-2 r y>2-y^{2}$ e, consequentemente,

$$
(y-r)^{2}=y^{2}-2 y r+r^{2}>y^{2}-2 y r>y^{2}+2-y^{2}=2 .
$$

Observação 2.4. Note que para encontrar $r$ nas demonstrações dos lemas 2.2 e 2.3 usamos o seguinte fato: dados $\mathrm{a}, \mathrm{b} \in \mathbb{Q}$ com $\mathrm{a}<\mathrm{b}$ existe $\mathrm{c} \in \mathbb{Q}$ tal que $\mathrm{a}<\mathrm{c}<\mathrm{b}$. $A$ demonstração de tal fato é bem simples: $c=\frac{a+b}{2} \in \mathbb{Q}$ satisfaz

$$
\frac{a+b}{2}<b, \text { pois } a+b<b+b=2 b
$$

$e$

$$
\frac{a+b}{2}>a, \text { pois } a+b>a+a=2 a \text {. }
$$

Lema 2.5. Se $x \in X$ e $y \in Y$ então $x<y$.

Demonstração: Suponha, por absurdo, que existem $x_{0} \in X$ e $y_{0} \in Y$ tal que $x_{0} \geq y_{0}$. Logo, temos $2<y_{0}^{2}=y_{0} y_{0} \leq x_{0} x_{0}=x_{0}^{2}$. Absurdo.

Proposição 2.6. Não existem $\sup X e \inf Y$.

Demonstração: Suponha, por absurdo, que existe $a=\sup X$. Pela tricotomia de $\mathbb{Q}$, existe apenas uma das três possibilidades abaixo para $a^{2}$ :

$$
\text { - } a^{2}<2
$$


- $a^{2}>2$

- $\mathrm{a}^{2}=2$

Afirmamos que não podemos ter $a^{2}<2$. De fato, $a=\sup X$ implica $a \geq 0$. Porém, $a \geq 0$ e $a^{2}<2$ implica que $a \in X$; consequentemente, a é o maior elemento de $X$. Absurdo, pois pelo lema 2.2, $\mathrm{X}$ não possui maior elemento.

Agora, afirmamos que não podemos ter $a^{2}>2$. De fato, $a=\sup X$ implica que $a \geq 0$. Porém, $a \geq 0$ e $a^{2}>2$ e implica que $a \in Y$. Pelo lema 2.3, $\exists r \in \mathbb{Q}$ tal que $a-r \in Y$.

Pelo lema 2.5, $a-r \geq x$, para todo $x \in X$. Absurdo, pois a $-r$ é cota superior de $X$ e $a-r<a=\sup X$.

Portanto, $a^{2}=2$. Absurdo, pois não existe $a \in \mathbb{Q} \operatorname{com} a^{2}=2$ (ver proposição 2.1).

Analogamente, mostra-se que não existe inf $Y$.

No que segue veremos que existe um corpo ordenado que contém $\mathbb{Q}$, para o qual qualquer subconjunto limitado superiormente admite supremo.

Se K é corpo ordenado, então, identificando $1 \in \mathbb{N}$ com o neutro multiplicativo de $K$ fica "natural" identificar $\mathbb{N}$ com um subconjunto de $K$; consequentemente, fica natural identificar $\mathbb{Z}$ e $\mathbb{Q}$ com subconjuntos de K. De fato, se K é corpo ordenado é possível considerar (ver, por exemplo, [5] e [14])

$$
\mathbb{N} \subset \mathbb{Z} \subset \mathbb{Q} \subset \mathrm{K} .
$$

Definição 2.7. Dizemos que um corpo ordenado $K$ é completo quando todo subconjunto não vazio limitado superiormente de $\mathrm{K}$ possui supremo em $\mathrm{K}$.

Exemplo 2.8. $\mathbb{Q}$ não é completo.

Adotaremos aqui o axioma fundamental da Análise Matemática (ver [10]).

Axioma: Existe um corpo ordenado completo, $\mathbb{R}$, chamado corpo dos números reais.

Observação 2.9. Como mencionado em [1] - seção 3.3, quaisquer dois corpos ordenados completos são isomorfos. Portanto, podemos considerar que tal $\mathbb{R}$ do axioma é único.

Definição 2.10. Dizemos que um subconjunto $Y \subset \mathbb{R}$ é enumerável se existe uma função $\mathrm{f}: \mathbb{N} \rightarrow \mathrm{Y}$ bijetora.

Uma propriedade importante de $\mathbb{R}$ é a seguinte: $\mathbb{R}$ não é enumerável (ver [10]). Como $\mathbb{R}=\mathbb{Q} \cup(\mathbb{R} \backslash \mathbb{Q})$ e $\mathbb{Q}$ é enumerável temos que $\mathbb{R} \backslash \mathbb{Q}$ não é enumerável (ver [10]).

O conjunto $\mathbb{R} \backslash \mathbb{Q}$ é chamado de conjunto dos números irracionais.

De maneira informal podemos dar a seguinte interpretação para o fato de $\mathbb{R} \backslash \mathbb{Q}$ não ser enumerável: existem mais números irracionais do que números racionais.

Então, quem são os irracionais? É fácil encontrar irracionais?

Denotemos $\sqrt{2}$ o número real tal que $(\sqrt{2})^{2}=2$. Pela proposição $2.1, \sqrt{2} \notin \mathbb{Q}$. 
A seguir, apresentaremos um número infinito enumerável de exemplos de números irracionais. Antes provaremos uma proposição que será muito útil.

Proposição 2.11. Sejam $p, m \in \mathbb{N}$ tais que $m d c(p, m)=1$. Se $n \in \mathbb{N}$ e $p \mid m n$ então $\mathrm{p} \mid \mathrm{n}$.

Demonstração: Se $p \mid m n$, então existe $k \in \mathbb{N}$ tal que $m n=p k$.

Agora, se $m d c(p, m)=1$ então, segue do algoritmo de Euclides (ver [5]), que existem $a, b \in \mathbb{N}$ tais que

$$
\mathrm{bp}-\mathrm{am}=1 .
$$

Multiplicando por $n$ ambos os lados da igualdade acima, obtemos

$$
\mathrm{n}=\mathrm{nbp}-\mathrm{nam} \text {. }
$$

Substituindo mn por pk nesta última igualdade, obtemos

$$
n=n b p-a p k=p(n b-a k) .
$$

Portanto, $p \mid n$.

Corolário 2.12. Se $p$ é primo e $p \mid m n$ então $p \mid m$ ou $p \mid n$.

Demonstração: Se $p \mid m$ o resultado é trivial. Suponha, então, que $p \nmid m$. Daí, como $p$ é primo temos que $m d c(p, m)=1$. Basta, então, aplicar a proposição acima.

Observação 2.13. Para mais resultados de divisibilidade ver, por exemplo, [9].

Proposição 2.14. Se $p \in \mathbb{N}$ é um número primo, então $\sqrt{\mathrm{p}}$ é irracional.

Demonstração: Suponhamos, por absurdo, que $\sqrt{p} \in \mathbb{Q}$. Então, existem $m, n \in \mathbb{N}$, com $\operatorname{mdc}(m, n)=1$, tais que $\sqrt{p}=\frac{m}{n}$. Segue que $p=\frac{m^{2}}{n^{2}}$ e, então, $m^{2}=p n^{2}$. Isto implica que $m^{2}$ é um múltiplo de $p$, ou seja, $p \mid m^{2}$. Portanto, pela proposição $2.11, p \mid m$, pois $p$ é primo.

Logo, existe $k \in \mathbb{N}$ tal que $m=k p$ e, por conseguinte, $m^{2}=k^{2} p^{2}$.

Sendo $\mathrm{m}^{2}=\mathrm{pn}^{2}$ e substituindo $\mathrm{m}^{2}$ por $\mathrm{k}^{2} \mathrm{p}^{2}$, temos $\mathrm{k}^{2} \mathrm{p}^{2}=\mathrm{pn}^{2}$. Logo, pela Lei do corte, $k^{2} p=n^{2}$; consequentemente, $p \mid n^{2}$. Portanto, novamente pela proposição $2.11, p \mid n$.

Como $\operatorname{mdc}(m, n)=1, p \mid m$ e $p \mid n$ temos que $p=1$. Absurdo, pois $p$ é primo.

Portanto, $\sqrt{\mathrm{p}} \in \mathbb{R} \backslash \mathbb{Q}$.

Os exemplos de números irracionais dados acima são obtidos usando "apenas" argumentos de Álgebra.

Nos dois próximos capítulos, mostraremos que $\pi$ e e são irracionais usando argumentos de Análise. 



\begin{tabular}{l|l|}
\hline CAPÍtulo \\
\cline { 2 - 3 }
\end{tabular}

\section{O número $\pi$}

Desde a Grécia antiga sabe-se que a razão entre o perímetro de uma circunferência e seu diâmetro é uma constante. Essa constante é representada pela letra grega $\pi$ (notação aparentemente introduzida pelo matemático William Jones (1675 - 1749)). Embora o número $\pi$ seja estudado (pelo menos) desde a antiga Grécia, apenas no século XVIII foi provada sua irracionalidade.

Em 1761, Johann Heinrich Lambert provou que $\pi$ é um número irracional. De fato, Lambert provou que se $0 \neq x \in \mathbb{Q}$ então $\tan (x)$ não é racional; note que $\tan \left(\frac{\pi}{4}\right)=1 \mathrm{e}$, portanto, $\frac{\pi}{4}$ não é racional.

Em 1947, Ivan Niven apresentou uma nova demonstração da irracionalidade de $\pi$ no artigo intitulado "A simple proof that $\pi$ is irrational" publicado no Bulletin of the American Mathematical Society (ver [12]).

Neste capítulo apresentaremos, com detalhes, a demonstração da irracionalidade de $\pi$ conforme encontrado em [6]. Tal demonstração utiliza ferramentas desenvolvidas num primeiro curso de cálculo diferencial e integral. 


\subsection{A irracionalidade de $\pi$}

Note que, se $\pi \in \mathbb{Q}$ então $\pi^{2} \in \mathbb{Q}$ (pois $\mathbb{Q}$ é fechado pela multiplicação). Logo, para mostrar que $\pi \notin \mathbb{Q}$ basta mostrar que $\pi^{2} \notin \mathbb{Q}$.

No que segue, mostraremos que $\pi^{2} \notin \mathbb{Q}$. Começamos com o seguinte lema:

Lema 3.1. Seja $a \in \mathbb{R}_{+}$. Então, $\lim _{n \rightarrow \infty} \frac{\pi a^{n}}{n !}=0$.

Demonstração: Considere a série $\sum_{n=0}^{\infty} \frac{\pi a^{n}}{n !}$. Temos

$$
\lim _{n \rightarrow \infty} \frac{\frac{\pi a^{n+1}}{(n+1) !}}{\frac{\pi a^{n}}{n !}}=\lim _{n \rightarrow \infty} \frac{a}{n+1}=0
$$

Como

$$
\lim _{n \rightarrow \infty} \frac{\frac{\pi a^{n+1}}{(n+1) !}}{\frac{\pi a^{n}}{n !}}<1
$$

temos, pelo teste da razão, que a série $\sum_{n=1}^{\infty} \frac{\pi a^{n}}{n !}$ converge. Daí,

$$
\lim _{n \rightarrow \infty} \frac{\pi a^{n}}{n !}=0
$$

Suponha, por absurdo, que $\pi^{2} \in \mathbb{Q}$. Logo, existem $a, b \in \mathbb{N}$, com $\operatorname{mdc}(a, b)=1$, tais que

$$
\pi^{2}=\frac{a}{b}
$$

Observação 3.3. Seja a dado por (3.2). Pelo lema 3.1, para $\mathrm{n}$ suficientemente grande temos $\frac{\pi \mathrm{a}^{\mathrm{n}}}{\mathrm{n} !}$ menor que 1. Mais precisamente, existe $\mathrm{n}_{0} \in \mathbb{N}$ tal que $\mathrm{n} \geq \mathrm{n}_{0} \Rightarrow \frac{\pi \mathrm{a}^{\mathrm{n}}}{\mathrm{n} !}<1$.

Agora, consideremos a função $f: \mathbb{R} \rightarrow \mathbb{R}$ definida por

$$
f(x)=\frac{x^{n}(1-x)^{n}}{n !}
$$

sendo $n$ algum $n \geq n_{0}$, com $n_{0}$ dado pela observação 3.3 . 
Note que a função $f$ é um polinômio de grau $2 n$ e que

$$
f(1-x)=\frac{(1-x)^{n}(1-(1-x))^{n}}{n !}=\frac{(1-x)^{n} x^{n}}{n !}=f(x), \quad \forall x \in \mathbb{R} .
$$

Vamos mostrar que $f^{(k)}(0)$ e $f^{(k)}(1)$ são números inteiros para qualquer $k=0,1,2, \cdots$. Para isso vamos utilizar a fórmula de Leibnitz:

$$
(g h)^{(k)}=\sum_{j=0}^{k}\left(\begin{array}{l}
k \\
j
\end{array}\right) g^{(j)} h^{(k-j)}, \forall k=0,1,2, \cdots
$$

Aplicando a fórmula acima à função $f$, obtemos

$$
f^{(k)}(x)=\frac{1}{n !} \sum_{j=0}^{k}\left(\begin{array}{l}
k \\
j
\end{array}\right)\left(\frac{d^{j}}{d x^{j}}\left(x^{n}\right)\right)\left(\frac{d^{(k-j)}}{d x^{(k-j)}}(1-x)^{n}\right) .
$$

Notemos que

$$
\left.\frac{d^{j}}{d x^{j}}\left(x^{n}\right)\right|_{x=0}=\left\{\begin{array}{cl}
0 & , \text { se } j<n \\
n ! & , \text { se } j=n \\
0, & \text { se } j>n
\end{array}\right.
$$

pois

$$
\left.\frac{d^{j}}{d x^{j}}\left(x^{n}\right)\right|_{x=0}=\left\{\begin{array}{cl}
n(n-1) \cdots(n-j+1) x^{n-j} & , \text { se } j \leq n \\
0 & , \text { se } j>n
\end{array} .\right.
$$

Uma vez que cada parcela apresenta um fator da forma $\left.\frac{d^{j}}{d x^{j}}\left(x^{\mathfrak{n}}\right)\right|_{x=0}, \operatorname{com} j=0,1, \cdots, k$, essas parcelas só não se anulam quando $j=n$. Agora, como $f^{(k)}(0)$ é escrito na forma

$$
f^{(k)}(0)=\frac{1}{n !} \sum_{j=0}^{k}\left(\begin{array}{l}
k \\
j
\end{array}\right)\left(\left.\frac{d^{j}}{d x^{j}}\left(x^{n}\right)\right|_{x=0}\right)\left(\left.\frac{d^{(k-j)}}{d x^{(k-j)}}(1-x)^{n}\right|_{x=0}\right) \text {, }
$$

temos o seguinte:

1. Se $k<n$ então $f^{(k)}(0)=0$; portanto, $f^{(k)}(0) \in \mathbb{Z}$ se $k<n$.

2. Se $k \geq n$ então

$$
\begin{aligned}
f^{(k)}(0) & =\frac{1}{n !}\left(\begin{array}{l}
k \\
n
\end{array}\right)\left(\left.\frac{d^{n}}{d x^{n}}\left(x^{n}\right)\right|_{x=0}\right)\left(\left.\frac{d^{(k-n)}}{d x^{(k-n)}}(1-x)^{n}\right|_{x=0}\right) \\
& =\frac{1}{n !}\left(\begin{array}{l}
k \\
n
\end{array}\right) n !\left(\left.\frac{d^{(k-n)}}{d x^{(k-n)}}(1-x)^{n}\right|_{x=0}\right) .
\end{aligned}
$$


Pela regra da cadeia, temos

$$
\frac{d^{l}}{d x^{l}}(1-x)^{n}=\left\{\begin{aligned}
(-1)^{l} n(n-1) \cdots(n+1-l)(1-x)^{n-l}, & \text { se } 1 \leq l<n \\
(-1)^{n} n !, & \text { se } l=n \\
0, & \text { se } l>n
\end{aligned}\right.
$$

Tomando $l=k-n$ temos

$$
\frac{d^{(k-n)}}{d x^{(k-n)}}(1-x)^{n}=\left\{\begin{aligned}
(-1)^{k-n} n(n-1) \cdots(2 n+1-k)(1-x)^{n-(k-n)}, & \text { se } n+1 \leq k<2 n \\
(-1)^{n} n !, & \text { se } k=2 n \\
0, & \text { se } k>2 n
\end{aligned}\right.
$$

Portanto, $f^{k}(0) \in \mathbb{Z}$ se $k \geq n$.

Falta mostrar que para todo $k=0,1,2, \cdots$, o número $f^{(k)}(1)$ é inteiro. Isso seguirá da relação $f(x)=f(1-x)$ e da Regra da Cadeia.

Derivando $k$ vezes ambos os lados da igualdade $f(x)=f(1-x)$, obtemos:

$$
\begin{aligned}
f^{\prime}(x) & =f^{\prime}(1-x)(-1)=(-1) f^{\prime}(1-x) \\
f^{\prime \prime}(x) & =(-1) f^{\prime \prime}(1-x)(-1)=(-1)^{2} f^{\prime}(1-x) \\
f^{\prime \prime \prime}(x) & =(-1)^{2} f^{\prime \prime \prime}(1-x)(-1)=(-1)^{3} f^{\prime \prime \prime}(1-x) \\
\vdots & \\
f^{(k)}(x) & =(-1)^{k} f^{(k)}(1-x) .
\end{aligned}
$$

Daí, $f^{(k)}(1)=(-1)^{k} f^{(k)}(1-1)=(-1)^{k} f^{(k)}(0) \in \mathbb{Z}$.

Considere, agora, a função $g: \mathbb{R} \rightarrow \mathbb{R}$ dada por

$$
g(x)=b^{n} \sum_{j=0}^{n}(-1)^{j} \pi^{2 n-2 j} f^{(2 j)}(x)=b^{n}\left[\pi^{2 n} f(x)-\pi^{2 n-2} f^{\prime \prime}(x)+\cdots+(-1)^{n} f^{(2 n)}(x)\right],
$$

sendo $n$ o mesmo da definição de $f$ em (3.4) e b dado por (3.2). Observemos que $b^{n} \pi^{2 n-2 j}=$ $b^{n}\left(\pi^{2}\right)^{n-j}=b^{n}\left(\frac{a}{b}\right)^{n-j}=b^{n} \frac{a^{n-j}}{b^{n-j}}=a^{n-j} b^{j}$ é um número inteiro sempre que $j \leq n$.

Logo, $g(0)$ e $g(1)$ são inteiros, pois

$$
g(0)=b^{0} a^{n} f(0)-b a^{n-1} f^{\prime \prime}(0)+\cdots+(-1)^{n} b^{n} f^{(2 n)}(0) \in \mathbb{Z}
$$

e

$$
g(1)=b^{0} a^{n} f(1)-b a^{n-1} f^{\prime \prime}(1)+\cdots+(-1)^{n} b^{n} f^{(2 n)}(1) \in \mathbb{Z} .
$$

Derivando duas vezes a função $g(x)$, temos:

$$
g^{\prime \prime}(x)=b^{n}\left[\pi^{2 n} f^{\prime \prime}(x)-\pi^{2 n-2} f^{(4)}(x)+\cdots+(-1)^{n-1} \pi^{2} f^{(2 n)}(x)+(-1)^{n} f^{(2 n+2)}(x)\right] .
$$


Como f é um polinômio de grau $2 n$ temos que a última parcela de equação anterior é igual a zero; logo, obtemos

$$
g^{\prime \prime}(x)=b^{n}\left[\pi^{2 n} f^{\prime \prime}(x)-\pi^{2 n-2} f^{(4)}(x)+\cdots+(-1)^{n-1} \pi^{2} f^{(2 n)}(x)\right] .
$$

Calculemos, agora $g^{\prime \prime}(x)+\pi^{2} g(x)$. Temos

$$
\begin{aligned}
g^{\prime \prime}(x)+\pi^{2} g(x)= & b^{n}\left[\pi^{2 n} f^{\prime \prime}(x)-\pi^{2 n-2} f^{(4)}(x)+\cdots+(-1)^{n-1} \pi^{2} f^{(2 n)}(x)\right]+ \\
& b^{n}\left[\pi^{2 n+2} f(x)-\pi^{2 n} f^{\prime \prime}(x)+\cdots+(-1)^{n} \pi^{2} f^{(2 n)}(x)\right] \\
= & b^{n}\left[\pi^{2 n+2} f(x)\right]=b^{n}\left[\pi^{2 n} \pi^{2} f(x)\right] \\
= & b^{n} \pi^{2} \frac{a^{n}}{b^{n}} f(x)=\pi^{2} a^{n} f(x) .
\end{aligned}
$$

Finalmente, definimos a função $h: \mathbb{R} \rightarrow \mathbb{R}$ dada por

$$
h(x)=g^{\prime}(x) \operatorname{sen}(\pi x)-\pi g(x) \cos (\pi x) .
$$

Derivando $\mathrm{h}$ temos

$$
\begin{aligned}
h^{\prime}(x) & =g^{\prime \prime}(x) \operatorname{sen}(\pi x)+\pi g^{\prime}(x) \cos (\pi x)-\pi\left[g^{\prime}(x) \cos (\pi x)+\pi g(x)(-\operatorname{sen}(\pi x))\right] \\
& =g^{\prime \prime}(x) \operatorname{sen}(\pi x)+\pi g^{\prime}(x) \cos (\pi x)-\pi g^{\prime}(x) \cos (\pi x)+\pi^{2} g(x) \operatorname{sen}(\pi x) \\
& =g^{\prime \prime}(x) \operatorname{sen}(\pi x)+\pi^{2} g(x) \operatorname{sen}(\pi x) \\
& =\left[g^{\prime \prime}(x)+\pi^{2} g(x)\right] \operatorname{sen}(\pi x) \\
& =\pi^{2} a^{n} f(x) \operatorname{sen}(\pi x) .
\end{aligned}
$$

Agora, pelo Teorema Fundamental do Cálculo, temos

$$
\int_{0}^{1} h^{\prime}(x) d x=h(1)-h(0)
$$

Daí,

$$
\begin{aligned}
\int_{0}^{1} \pi^{2} a^{n} f(x) \operatorname{sen}(\pi x) d x & =g^{\prime}(1) \operatorname{sen} \pi-\pi g(1) \cos \pi-\left[g^{\prime}(0) \operatorname{sen} 0-\pi g(0) \cos 0\right] \\
& =\pi g(1)+\pi g(0)=\pi(g(1)+g(0))
\end{aligned}
$$

Assim,

$$
\pi^{2} \int_{0}^{1} a^{n} f(x) \operatorname{sen}(\pi x) d x=\pi(g(1)+g(0))
$$

Logo,

$$
\pi \int_{0}^{1} a^{n} f(x) \operatorname{sen}(\pi x) d x=(g(1)+g(0)) \in \mathbb{Z},
$$

isto é, $\pi \int_{0}^{1} a^{n} f(x) \operatorname{sen}(\pi x) d x$ é um número inteiro. 
Vamos, agora, limitar o valor de

$$
\pi \int_{0}^{1} a^{n} f(x) \operatorname{sen}(\pi x) d x
$$

Temos o seguinte:

Lema 3.5. Seja $\mathrm{f}$ dada por (3.4). Se $0<x<1$ então $0<\mathrm{f}(\mathrm{x})<\frac{1}{\mathrm{n} !}$.

Demonstração: Se $0<x<1$ então $0<x^{n}<1$. Além disso, se $0<x<1$, então $-1<-x<0$; consequentemente, $0<1-x<1$. Daí, $0<(1-x)^{n}<1$.

Logo, para $0<x<1$ temos

$$
0<x^{\mathrm{n}}(1-x)^{\mathrm{n}}<1
$$

Agora, dividindo (3.6) por $n$ ! obtemos

$$
0<\frac{x^{n}(1-x)^{n}}{n !}<\frac{1}{n !}
$$

Portanto, $0<f(x)<\frac{1}{n !}$, se $x \in(0,1)$.

Note que para $x \in(0,1)$ temos $0<\operatorname{sen}(\pi x)<1$. Disto e do lema 3.5, segue que

$$
0<f(x) \operatorname{sen}(\pi x)<\frac{1}{n !} .
$$

Daí, para $x \in(0,1)$ temos

$$
0<\pi a^{n} f(x) \operatorname{sen}(\pi x)<\frac{\pi a^{n}}{n !} .
$$

Integrando as funções acima, obtemos

$$
0<\int_{0}^{1} \pi a^{n} f(x) \operatorname{sen}(\pi x) d x<\int_{0}^{1} \frac{\pi a^{n}}{n !} d x=\left.\frac{\pi a^{n}}{n !} x\right|_{0} ^{1}=\frac{\pi a^{n}}{n !}<1
$$

para $n$ suficientemente grande. Absurdo, pois $\pi \int_{0}^{1} a^{n} f(x) \operatorname{sen}(\pi x) d x$ é um inteiro e não existe inteiro entre 0 e 1.

O absurdo ocorreu ao supor que $\pi^{2}$ é racional. Portanto, $\pi^{2}$ é irracional; consequentemente, $\pi$ é irracional. 


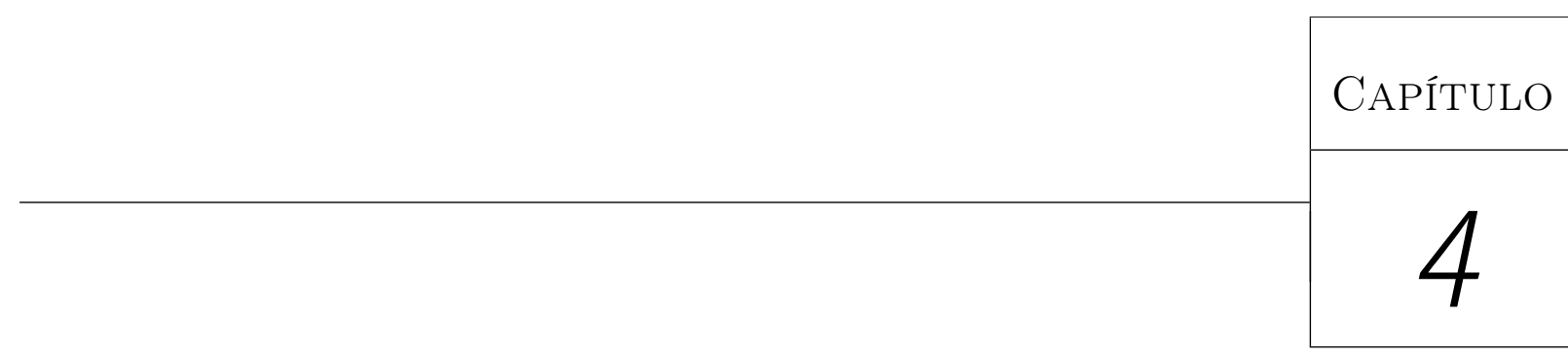

\section{O número e}

Não se sabe a data exata do surgimento do número e, segundo Maor em [11]:

As origens de e não são tão claras, elas parecem recuar ao século XVI, quando se percebeu que a expressão $(1+1 / n)^{n}$, que aparecia na fórmula dos juros compostos, tendia a um certo limite - cerca de 2,71828 - à medida que $\mathrm{n}$ aumenta. Assim tornou-se o primeiro número a ser definido por um processo de limite, $e=\lim _{n \rightarrow \infty}(1+1 / n)^{n}$.

O número e aparece de forma espontânea em situações práticas (veja, por exemplo, [11]). Vejamos um exemplo utilizando juros compostos.

Um capital $\mathrm{C}_{0}$ é aplicado a uma taxa de $k \%$ ao longo de um ano. Vamos imaginar que o juro seja incorporado apenas ao final do ano. Neste caso teríamos um montante equivalente a $C_{0}+\frac{k}{100}$. $C_{0}$. Isto significa que o capital ficou estagnado durante todo o ano e apenas na passagem para o ano seguinte sofreu o acréscimo de $k \%$.

Parece mais razoável que esta taxa de $k \%$ seja distribuída ao longo do ano.

Agora, vamos imaginar que a taxa seja aplicada a cada 6 meses. Neste caso, teríamos até a metade do ano uma taxa de $\frac{k}{2} \%$, o que equivale a dizer que no primeiro semestre o capital passaria a valer $C_{0}+\frac{k}{200}$. $C_{0}$. Este capital no final do segundo semestre sofreria um 
acréscimo de $\frac{k}{2} \%$ e seu valor se tornaria $C_{0}+\frac{k}{200} \cdot C_{0}+\frac{k}{200} \cdot\left(C_{0}+\frac{k}{200} \cdot C_{0}\right)$, o que equivale $\mathrm{a}\left(\mathrm{C}_{0}+\frac{\mathrm{k}}{200} \cdot \mathrm{C}_{0}\right) \cdot\left(1+\frac{\mathrm{k}}{200}\right)$; isto é, $\mathrm{C}_{0} \cdot\left(1+\frac{\mathrm{k}}{200}\right) \cdot\left(1+\frac{\mathrm{k}}{200}\right)$. Assim, se a taxa for distribuída a cada 6 meses, no final de um ano o capital passaria a ser $C_{0} \cdot\left(1+\frac{k}{200}\right)^{2}$.

Agora, vamos distribuir esta taxa a cada trimestre. No final do primeiro trimestre teríamos $\mathrm{C}_{0}+\frac{\mathrm{k}}{400} \cdot \mathrm{C}_{0}$, ou ainda, $\mathrm{C}_{0}\left(1+\frac{\mathrm{k}}{400}\right)$. No final do segundo trimestre o capital seria igual a $C_{0}\left(1+\frac{k}{400}\right)^{2}$. No final do terceiro trimestre teríamos $C_{0}\left(1+\frac{k}{400}\right)^{3}$ e finalmente no término do ano o capital passaria a ser $C_{0}\left(1+\frac{k}{400}\right)^{4}$.

Repetindo o raciocínio anterior, se imaginarmos a taxa distribuída uniformemente ao longo do ano dividido em $n$ períodos, no final do ano teríamos $C=C_{0}\left(1+\frac{k}{n \times 100}\right)^{n}$.

Surge, então, uma pergunta natural: a medida que $n$ tende ao infinito, qual é o valor $\operatorname{de}\left(1+\frac{k}{n \times 100}\right)^{n} ?$

Simbolicamente, quanto é $\lim _{n \rightarrow \infty}\left(1+\frac{k}{n \times 100}\right)^{n}$ ?

Vamos utilizar alguns artifícios de cálculo para determinarmos o limite acima.

Consideremos inicialmente que $\frac{\mathrm{k}}{\mathrm{n} \times 100}=\frac{1}{\mathrm{~m}}$ ou, equivalentemente, $\mathrm{n} \times 100=\mathrm{m} \times \mathrm{k}$; note que $n \rightarrow \infty$ se, e somente se, $m \rightarrow \infty$.

Assim, podemos escrever

$$
\left(1+\frac{k}{n \times 100}\right)^{n}=\left(1+\frac{1}{m}\right)^{m \frac{k}{100}}=\left[\left(1+\frac{1}{m}\right)^{m}\right]^{\frac{k}{100}}
$$

Então,

$$
\lim _{n \rightarrow \infty}\left(1+\frac{k}{n \times 100}\right)^{n}=\lim _{m \rightarrow \infty}\left[\left(1+\frac{1}{m}\right)^{m}\right]^{\frac{k}{100}}=\left[\lim _{m \rightarrow \infty}\left(1+\frac{1}{m}\right)^{m}\right]^{\frac{k}{100}}
$$

Logo, devemos verificar se existe $\lim _{m \rightarrow \infty}\left(1+\frac{1}{m}\right)^{m}$.

Antes vamos mostrar que existe $\lim _{n \rightarrow \infty} S_{n}$, sendo

$$
S_{n}=1+\frac{1}{1 !}+\frac{1}{2 !}+\cdots+\frac{1}{n !}, \quad \text { com } \quad n=1,2,3, \cdots
$$


Note que $S_{n}>2$ para todo $n \in \mathbb{N}$.

Vamos mostrar que a sequência é monótona, crescente e limitada, garantindo, assim, que ela converge para um limite $S$.

Observa-se facilmente que a sequência é monótona, pois como $n$ é natural todos os termos são positivos e a soma aumenta a cada termo adicional; ou seja, $S_{\mathfrak{n}}<S_{n+1}$. Então, a sequência é crescente.

Note que, para $n \geq 3$, temos $n !=1 \cdot 2.3 .4 \cdots n>1.2 .2 \cdots=2^{n-1}$. Assim, podemos escrever

$$
S_{n}<1+1+\frac{1}{2}+\frac{1}{2^{2}}+\cdots+\frac{1}{2^{n-1}}
$$

Vamos, agora, limitar o valor da soma

$$
1+1+\frac{1}{2}+\frac{1}{2^{2}}+\cdots+\frac{1}{2^{n-1}}
$$

Observe que a partir do segundo termo de (4.2), temos uma progressão geométrica com primeiro termo igual a 1 e razão igual a $\frac{1}{2}$. Aplicando a fórmula para calcular a soma de uma PG infinita, temos

$$
1+1+\frac{1}{2}+\frac{1}{2^{2}}+\cdots+\frac{1}{2^{n-1}}+\cdots=1+\frac{1}{1-\frac{1}{2}}=1+\frac{1}{\frac{1}{2}}=1+2=3
$$

Logo $S_{n}<3$ para todo $n \in \mathbb{N}$. Assim, mostramos que a sequência $\left(S_{n}\right)_{n \geq 1}$ é limitada superiormente por 3, ou seja, seus valores nunca excedem 3.

Portanto, como toda sequência monótona limitada é convergente (ver [10]), temos que $\left(S_{n}\right)_{n \geq 1}$ converge para um limite $S$ e esse limite está entre 2 e 3.

Agora, vamos mostrar que $T_{n}=\left(1+\frac{1}{n}\right)^{n}$ converge para o mesmo limite de $S_{n}$; isto é, vamos mostrar que $\lim _{n \rightarrow \infty} T_{n}=S$.

Utilizando o binômio de Newton, observamos que

$$
(a+b)^{n}=\left(\begin{array}{l}
n \\
0
\end{array}\right) a^{n} b^{0}+\left(\begin{array}{l}
n \\
1
\end{array}\right) a^{n-1} b^{1}+\left(\begin{array}{l}
n \\
2
\end{array}\right) a^{n-2} b^{2}+\cdots+\left(\begin{array}{l}
n \\
n
\end{array}\right) a^{0} b^{n}
$$


Então, para $a=1$ e $b=\frac{1}{n}$, temos:

$$
\begin{aligned}
T_{n} & =\left(1+\frac{1}{n}\right)^{n} \\
& =\left(\begin{array}{l}
n \\
0
\end{array}\right) 1^{n}\left(\frac{1}{n}\right)^{0}+\left(\begin{array}{l}
n \\
1
\end{array}\right) 1^{n-1}\left(\frac{1}{n}\right)^{1}+\left(\begin{array}{l}
n \\
2
\end{array}\right) 1^{n-2}\left(\frac{1}{n}\right)^{2}+\cdots+\left(\begin{array}{l}
n \\
n
\end{array}\right) 1^{0}\left(\frac{1}{n}\right)^{n} \\
& =1+n \cdot \frac{1}{n}+\frac{n(n-1)}{2 !} \cdot \frac{1}{n^{2}}+\cdots+\frac{n(n-1)(n-2) \cdots \cdot 1}{n !} \cdot \frac{1}{n^{n}} \\
& =1+1+\left(1-\frac{1}{n}\right) \cdot \frac{1}{2 !}+\cdots+\left(1-\frac{1}{n}\right) \cdot\left(1-\frac{2}{n}\right) \cdots\left(1-\frac{n-1}{n}\right) \cdot \frac{1}{n !}
\end{aligned}
$$

Observe que as expressões dentro de cada parênteses são menores do que 1 ; assim $T_{n} \leq S_{n}$. Então, podemos concluir que a sequência $\left(T_{n}\right)$ é limitada superiormente. Afirmamos que $\left(T_{n}\right)$ é monótona crescente. De fato,

$$
T_{n}=1+1+\left(1-\frac{1}{n}\right) \cdot \frac{1}{2 !}+\cdots+\left(1-\frac{1}{n}\right) \cdot\left(1-\frac{2}{n}\right) \cdots\left(1-\frac{n-1}{n}\right) \cdot \frac{1}{n !}
$$

e

$$
\begin{aligned}
T_{n+1} & =1+1+\left(1-\frac{1}{n+1}\right) \cdot \frac{1}{2 !}+\left(1-\frac{1}{n+1}\right) \cdot\left(1-\frac{2}{n+1}\right) \cdot \frac{1}{3 !}+\cdots \\
& +\left(1-\frac{1}{n+1}\right) \cdot\left(1-\frac{2}{n+1}\right) \cdots\left(1-\frac{n-1}{n+1}\right) \cdot \frac{1}{n !} \\
& +\left(1-\frac{1}{n+1}\right) \cdot\left(1-\frac{2}{n+1}\right) \cdots\left(1-\frac{n-1}{n+1}\right) \cdot\left(1-\frac{n}{n+1}\right) \cdot \frac{1}{(n+1) !}
\end{aligned}
$$

Como a última parcela de $\mathrm{T}_{\mathrm{n}+1}$ é maior que zero, temos

$$
\begin{aligned}
\mathrm{T}_{\mathrm{n}+1} & >1+1+\left(1-\frac{1}{\mathrm{n}+1}\right) \cdot \frac{1}{2 !}+\left(1-\frac{1}{n+1}\right) \cdot\left(1-\frac{2}{n+1}\right) \cdot \frac{1}{3 !}+\cdots \\
& +\left(1-\frac{1}{n+1}\right) \cdot\left(1-\frac{2}{n+1}\right) \cdots\left(1-\frac{n-1}{n+1}\right) \cdot \frac{1}{n !}
\end{aligned}
$$

Agora,

$$
\begin{aligned}
\mathrm{n}<\mathrm{n}+1 & \Leftrightarrow \frac{1}{\mathrm{n}}>\frac{1}{\mathrm{n}+1} \Leftrightarrow-\frac{1}{\mathrm{n}}<-\frac{1}{\mathrm{n}+1} \Leftrightarrow-\frac{\mathrm{k}}{\mathrm{n}}<-\frac{\mathrm{k}}{\mathrm{n}+1}, \forall \mathrm{k}>0 \\
& \Leftrightarrow 1-\frac{\mathrm{k}}{\mathrm{n}}<1-\frac{\mathrm{k}}{\mathrm{n}+1}, \forall \mathrm{k}>0 .
\end{aligned}
$$


Em particular, tomando $k=1,2, \cdots, n-1$, temos

$$
\begin{aligned}
T_{n+1} & >1+1+\left(1-\frac{1}{n+1}\right) \cdot \frac{1}{2 !}+\left(1-\frac{1}{n+1}\right) \cdot\left(1-\frac{2}{n+1}\right) \cdot \frac{1}{3 !}+\cdots \\
& +\left(1-\frac{1}{n+1}\right) \cdot\left(1-\frac{2}{n+1}\right) \cdots\left(1-\frac{n-1}{n+1}\right) \cdot \frac{1}{n !} \\
& >1+1+\left(1-\frac{1}{n}\right) \cdot \frac{1}{2 !}+\cdots+\left(1-\frac{n-1}{n}\right) \cdot \frac{1}{n !}=T_{n} .
\end{aligned}
$$

Logo, $T_{n+1}>T_{n}$.

Portanto, como $\left(T_{n}\right)$ é uma seqüência crescente e limitada temos que $\left(T_{n}\right)$ converge para um limite, digamos $T$.

Falta mostrar que $S=T$. Para isso, vamos mostrar que $S \geq T$ e $S \leq T$.

Para mostrar que $S \geq T$ é simples, pois já provamos que $S_{n} \geq T_{n}$, para todo $n$. Então,

$$
S=\lim _{n \rightarrow \infty} S_{n} \geq \lim _{n \rightarrow \infty} T_{n}=T \text {. }
$$

Vamos mostrar, agora, que $S \leq T$. Fixe $m$ e seja $n>m$. Vamos escrever os primeiros $m+1$ termos de $T_{n}$ :

$$
R_{m, n}=1+1+\left(1-\frac{1}{n}\right) \cdot \frac{1}{2 !}+\cdots+\left(1-\frac{1}{n}\right) \cdot\left(1-\frac{2}{n}\right) \cdot\left(1-\frac{m-1}{n}\right) \cdot \frac{1}{m !} .
$$

Note que, como $m<n$ e todos os termos de $\left(T_{n}\right)$ são positivos, $R_{m, n}<T_{n}$. Note também que como $m$ é fixo temos

$$
\lim _{n \rightarrow \infty} R_{m, n}=S_{m} \text {. }
$$

Agora, como $R_{m, n}<T_{n}$, temos

$$
S_{m}=\lim _{n \rightarrow \infty} R_{m, n} \leq \lim _{n \rightarrow \infty} T_{n}=T .
$$

Logo,

$$
\mathrm{S}=\lim _{\mathrm{m} \rightarrow \infty} \mathrm{S}_{\mathrm{m}} \leq \mathrm{T}
$$

Portanto, $\mathrm{S}=\mathrm{T}$.

Definição 4.3. Definimos

$$
e=\lim _{n \rightarrow \infty}\left(1-\frac{1}{n}\right)^{n}
$$

equivalentemente,

$$
e=\sum_{i=0}^{\infty} \frac{1}{n !}
$$


Finalmente, estamos prontos para responder nossa pergunta: a medida que $n$ aumenta, qual é o valor de $\left(1+\frac{k}{n \times 100}\right)^{n}$ ?

Resposta: $e^{\frac{k}{100}}$.

Portanto, um capital $\mathrm{C}_{0}$ aplicado a uma taxa de $k \%$ ao longo de um ano passará a ser $\mathrm{C}_{0} \mathrm{e}^{\frac{\mathrm{k}}{100}}$ ao final da aplicação.

Observação 4.4. Evidentemente, $2<\sum_{n=0}^{\infty} \frac{1}{n !}$. Além disso,

$$
\sum_{n=0}^{\infty} \frac{1}{n !}=1+1+\frac{1}{2}+\frac{1}{6}+\sum_{n=4}^{\infty} \frac{1}{n !}
$$

e, como em (4.1),

$$
\sum_{n=0}^{\infty} \frac{1}{n !} \leq 1+1+\frac{1}{2}+\frac{1}{6}+\sum_{n=4}^{\infty} \frac{1}{2^{n-1}} .
$$

Agora, aplicando a fórmula para calcular a soma de uma PG infinita, temos

$$
\sum_{n=4}^{\infty} \frac{1}{2^{n-1}}=\frac{1}{4}
$$

$\log 0$,

$$
\sum_{n=0}^{\infty} \frac{1}{n !} \leq 1+1+\frac{1}{2}+\frac{1}{6}+\frac{1}{4}<1+1+\frac{1}{2}+\frac{1}{4}+\frac{1}{4}=3
$$

Portanto, $2<\mathrm{e}<3$.

\subsection{A irracionalidade do número e}

Acreditamos que a prova da irracionalidade do número de Euler, e, possa ser compreendida por um aluno de educação básica porque utiliza apenas argumentos simples de frações, fatorial, sequências e manipulações algébricas básicas.

Nesta seção apresentaremos uma demonstração detalhada da irracionalidade de $e$, baseada naquela apresentada em [11].

Suponha, por absurdo, que e é um número racional, ou seja, $e=\frac{p}{q}$, com $p$ e $q$ naturais. Podemos assumir que $\operatorname{mdc}(p, q)=1$. Como $2<e<3$ temos que e $\notin \mathbb{N}$; consequentemente, $q \geq 2$. Sabemos que

$$
e=\sum_{i=0}^{\infty} \frac{1}{n !}=\frac{1}{0 !}+\frac{1}{1 !}+\frac{1}{2 !}+\frac{1}{3 !}+\cdots
$$


Assim,

$$
\frac{p}{q}=e=1+1+\frac{1}{2 !}+\frac{1}{3 !}+\cdots+\frac{1}{(q-1) !}+\frac{1}{q !}+\frac{1}{(q+1) !}+\cdots+\frac{1}{n !}+\cdots
$$

Multiplicando a igualdade acima por q!, obtemos

$$
\begin{aligned}
\frac{p}{q} \cdot q ! & =1 \cdot q !+1 \cdot q !+\frac{1}{2 !} \cdot q !+\frac{1}{3 !} \cdot q !+\cdots+\frac{1}{(q-1) !} \cdot q !+\frac{1}{q !} \cdot q !+\frac{1}{(q+1) !} \cdot q ! \\
& +\cdots+\frac{1}{n !} \cdot q !+\cdots
\end{aligned}
$$

Daí,

$$
\begin{aligned}
\frac{p}{q} \cdot q \cdot(q-1) ! & =q !+q !+\frac{1}{2 !} \cdot 1 \cdot 2 \cdot 3 \ldots q+\frac{1}{3 !} \cdot 1 \cdot 2 \cdot 3 \ldots q+\cdots+\frac{1}{(q-1) !} \cdot q \cdot(q-1) !+\frac{1}{q !} \cdot q ! \\
& +\frac{1}{(q+1) !} \cdot q !+\cdots+\frac{1}{n !} \cdot q !+\cdots
\end{aligned}
$$

e, consequentemente,

$$
\begin{aligned}
p .(q-1) ! & =q !+q !+3.4 \ldots q+4.5 \ldots q+\cdots+q+1+\frac{1}{(q+1)} \\
& +\frac{1}{(q+2)(q+1)}+\cdots+\frac{1}{n !} q !+\cdots
\end{aligned}
$$

Logo,

$$
\begin{aligned}
p .(q-1) !-(q !+q !+ & 3.4 \ldots q+4.5 \ldots q+\cdots+q+1) \\
& =\frac{1}{(q+1)}+\frac{1}{(q+2)(q+1)}+\cdots+\frac{1}{n !} q !+\cdots .
\end{aligned}
$$

Observe que no lado esquerdo da igualdade (4.5) as parcelas são números inteiros, enquanto que no lado direito as parcelas são frações; além disso, como em (4.5) tem-se $q \geq 2$, obtemos

$$
\begin{aligned}
\frac{1}{q+1} & \leq \frac{1}{3} \\
\frac{1}{(q+2) \cdot(q+1)} & \leq \frac{1}{3} \cdot \frac{1}{3}=\frac{1}{3^{2}} \\
\vdots & \leq \frac{1}{3} \cdots \frac{1}{3}=\frac{1}{3^{n-q}} .
\end{aligned}
$$


Logo, em (4.5), temos

$$
\frac{1}{q+1}+\frac{1}{(q+2) \cdot(q+1)}+\cdots+\frac{1}{n !} \cdot q !+\cdots \leq \frac{1}{3}+\frac{1}{3^{2}}+\cdots+\frac{1}{3^{n-q}}+\cdots
$$

Observe que

$$
\frac{1}{3}+\frac{1}{3^{2}}+\cdots+\frac{1}{3^{n-q}}+\cdots
$$

é uma progressão geométrica, sendo que o primeiro termo é $\frac{1}{3}$ e a razão também é $\frac{1}{3} ; \log$,

$$
\frac{1}{3}+\frac{1}{3^{2}}+\cdots+\frac{1}{3^{n-q}}+\cdots=\frac{\frac{1}{3}}{1-\frac{1}{3}}=\frac{\frac{1}{3}}{\frac{2}{3}}=\frac{1}{2}
$$

Portanto, segue de (4.5), (4.6) e (4.7) que

$$
p(q-1) !-[q !+\cdots+1] \leq \frac{1}{2} .
$$

Absurdo, pois não existe número inteiro entre 0 e $\frac{1}{2}$. O absurdo ocorreu ao supor que e é número racional; portanto, e é um número irracional. 


\begin{tabular}{|c|c|}
\hline \multirow{2}{*}{ CAPÍTULO } \\
\cline { 2 - 2 } & 5 \\
\hline
\end{tabular}

\section{O número e: possibilidade para a sala de aula}

Acreditamos que seja possível discutir a demonstração da irracionalidade de e com os alunos do ensino médio. De fato, podemos definir e, e verificar sua irracionalidade, utilizando apenas propriedades de sequências e séries.

Neste capítulo optamos por utilizar uma linguagem menos técnica e mais intuitiva do que aquela apresentada no capítulo 4.

Acreditamos que o texto que apresentaremos a seguir possa ser utilizado para formar um conjunto de aulas para o Ensino Médio, bem como, para alunos de cursos de Licenciatura em Matemática.

\subsection{Surgimento do número e}

Segundo Maor em [11], não se sabe a data exata do surgimento do número e: 
As origens de e não são tão claras, elas parecem recuar ao século XVI, quando se percebeu que a expressão $(1+1 / n)^{n}$, que aparecia na fórmula dos juros compostos, tendia a um certo limite - cerca de 2,71828

- à medida que $\mathrm{n}$ aumenta. Assim tornou-se o primeiro número a ser definido por um processo de limite, $e=\lim _{n \rightarrow \infty}(1+1 / n)^{n}$.

Vejamos:

Um capital de $R \$ 100,00$ é aplicado a uma taxa de $15 \%$ ao longo de um ano. Vamos imaginar que o juro seja incorporado apenas ao final do ano. Neste caso teríamos um montante equivalente a

$$
100+\frac{15}{100} 100, \quad \text { isto é, } R \$ 115,00 .
$$

Isto significa que o capital ficou estagnado durante todo o ano e apenas na passagem para o ano seguinte sofreu o acréscimo de $15 \%$.

Note que se desejássemos sacar os $R \$ 100,00$ ao final de 6 meses não receberíamos nada de juros. Então, parece mais razoável que ao sacar os $\mathrm{R} \$ 100,00$ ao final de 6 meses recebessemos $\frac{15}{2} \%$ de juros. Dessa forma, ao final de 6 meses o montante passaria a valer $100+\frac{15}{200} 100$, isto é, $R \$ 107,50$ ao invés de $R \$ 100,00$. Logo, ao deixar este montante aplicado até o final do ano ele sofreria um acréscimo de $\frac{15}{2} \%$ e seu valor passaria a ser $100+\frac{15}{200} 100+\frac{15}{200}\left(100+\frac{15}{200} 100\right)$, o que equivale a $\left(100+\frac{15}{200} 100\right)\left(1+\frac{15}{200}\right) ;$ isto é, $100\left(1+\frac{15}{200}\right)\left(1+\frac{15}{200}\right)$. Assim, se a taxa for distribuída a cada 6 meses, no final de um ano o capital passaria a ser $100\left(1+\frac{15}{200}\right)^{2}$.

Podemos aplicar o raciocínio acima para verificar o rendimento do capital a cada 4 meses, isto é, a cada trimestre. No final do primeiro trimestre deveríamos ter $100+$ $\frac{15}{400} 100$, ou ainda, $100\left(1+\frac{15}{400}\right)$. No final do segundo trimestre o capital deveria ser igual a $100\left(1+\frac{15}{400}\right)^{2}$. No final do terceiro trimestre deveríamos ter $100\left(1+\frac{15}{400}\right)^{3}$ e, finalmente, no término do ano o capital passaria a ser $100\left(1+\frac{15}{400}\right)^{4}$.

Repetindo o raciocínio anterior, se imaginarmos a taxa distribuída uniformemente ao longo do ano dividido em $n$ períodos, no final do ano teríamos

$$
C=100\left(1+\frac{15}{100 n}\right)^{n} \text {. }
$$

Aparentemente, os bancos usam $n=12$. Verifique! 
Surge, então, uma pergunta natural:

A medida que $n$ aumenta, qual o valor de $\left(1+\frac{15}{100 n}\right)^{n}$ ?

Podemos simplificar nossa pergunta fazendo $\frac{15}{100 \mathrm{n}}=\frac{1}{\mathrm{~m}}$ ou, equivalentemente, $100 \mathrm{n}=$ $15 \mathrm{~m}$; note que $\mathrm{n}$ aumenta se, e somente se, $m$ aumenta.

Assim, podemos escrever

$$
\left(1+\frac{15}{100 n}\right)^{n}=\left(1+\frac{1}{m}\right)^{\frac{15 m}{100}}=\left[\left(1+\frac{1}{m}\right)^{\frac{m}{100}} .\right.
$$

Nossa pergunta, então, é equivalente a seguinte:

A medida que $m$ aumenta, qual o valor de $\left(1+\frac{1}{m}\right)^{m}$ ?

Antes de responder a pergunta acima, vamos estudar o comportamento da sequência $\left(S_{n}\right)$, cujos termos são dados por

$$
S_{n}=\frac{1}{0 !}+\frac{1}{1 !}+\frac{1}{2 !}+\cdots+\frac{1}{n !}
$$

Note que $1 \leq S_{n}<S_{n+1}$ para todo $n$.

Também, note que podemos escrever $S_{n}$ na forma

$$
S_{n}=\sum_{k=0}^{n} \frac{1}{k !}
$$

Logo, quando fazemos $n$ aumentar indefinidamente (isto, $n$ ir para o infinito) queremos calcular

$$
\sum_{k=0}^{\infty} \frac{1}{k !}
$$

isto é, queremos encontrar $S \in \mathbb{R}$ tal que

$$
S=\sum_{k=0}^{\infty} \frac{1}{k !}
$$

Note que, para $n \geq 3$, temos $n !=1.2 .3 \cdots n>1.2 .2 \cdots 2=2^{n-1}$. Assim, podemos escrever

$$
S_{n}<1+1+\frac{1}{2}+\frac{1}{2^{2}}+\cdots+\frac{1}{2^{n-1}}=1+\sum_{k=0}^{n-1} \frac{1}{2^{k}} .
$$

Daí,

$$
S_{n}<1+\sum_{k=0}^{n-1} \frac{1}{2^{k}}<1+\sum_{k=0}^{\infty} \frac{1}{2^{k}}
$$


Como $\sum_{\mathrm{k}=0}^{\infty} \frac{1}{2^{\mathrm{k}}}=1+\frac{1}{2}+\frac{1}{2^{2}}+\cdots+\frac{1}{2^{\mathrm{k}}}+\cdots$ é uma Progressão Geométrica com primeiro termo igual a 1 e razão igual a $\frac{1}{2}$, podemos aplicar a fórmula para calcular a soma de uma PG infinita e, então, obtemos

$$
\sum_{k=0}^{\infty} \frac{1}{2^{k}}=\frac{1}{1-\frac{1}{2}}=\frac{1}{\frac{1}{2}}=2
$$

Logo, para cada $n$ temos $S_{n}<3$. Assim, mostramos que a sequência $\left(S_{n}\right)$ é limitada superiormente por 3 , ou seja, seus valores nunca excedem 3 .

Portanto, como $S_{n}<S_{n+1}$ e $S_{n}$ está entre 1 e 3 , para cada $n \geq 0$, deve existir um $S \in \mathbb{R}$, com $1 \leq S \leq 3$, tal que

$$
S=\sum_{k=0}^{\infty} \frac{1}{k !}
$$

Abaixo, segue uma tabela que nos sugere uma aproximação para a soma S:

Tabela 5.1: Aproximação de $S$

\begin{tabular}{|c|l|c|}
\hline$n$ & $\sum_{k=0}^{n} \frac{1}{k !}$ & $S_{n}$ \\
\hline 0 & $\sum_{k=0}^{0} \frac{1}{k !}=\frac{1}{1}$ & 1 \\
\hline 1 & $\sum_{k=0}^{1} \frac{1}{k !}=\frac{1}{1}+\frac{1}{1}$ & 2 \\
\hline 2 & $\sum_{k=0}^{2} \frac{1}{k !}=\frac{1}{1}+\frac{1}{1}+\frac{1}{2}$ & 2,5 \\
\hline 3 & $\sum_{k=0}^{3} \frac{1}{k !}=\frac{1}{1}+\frac{1}{1}+\frac{1}{2}+\frac{1}{6}$ & $\approx 2,666666$ \\
\hline 4 & $\sum_{k=0}^{4} \frac{1}{k !}=\frac{1}{1}+\frac{1}{1}+\frac{1}{2}+\frac{1}{6}+\frac{1}{24}$ & $\approx 2,708332$ \\
\hline 5 & $\sum_{k=0}^{5} \frac{1}{k !}=\frac{1}{1}+\frac{1}{1}+\frac{1}{2}+\frac{1}{6}+\frac{1}{24}+\frac{1}{120}$ & $\approx 2,716665$ \\
\hline 6 & $\sum_{k=0}^{6} \frac{1}{k !}=\frac{1}{1}+\frac{1}{1}+\frac{1}{2}+\frac{1}{6}+\frac{1}{24}+\frac{1}{120}+\frac{1}{720}$ & $\approx 2,718053$ \\
\hline 7 & $\sum_{k=0}^{7} \frac{1}{k !}=\frac{1}{1}+\frac{1}{1}+\frac{1}{2}+\frac{1}{6}+\frac{1}{24}+\frac{1}{120}+\frac{1}{720}+\frac{1}{5040}$ & $\approx 2,718251$ \\
\hline
\end{tabular}

Pela tabela 1 podemos indicar que $S$ é aproximadamente 2, 718 . 
Agora, vamos mostrar que quando $n$ aumenta $T_{n}=\left(1+\frac{1}{n}\right)^{n}$ fica perto de $S$.

Recordemos que o binômio de Newton se escreve como

$$
(a+b)^{n}=\left(\begin{array}{l}
n \\
0
\end{array}\right) a^{n} b^{0}+\left(\begin{array}{l}
n \\
1
\end{array}\right) a^{n-1} b^{1}+\left(\begin{array}{l}
n \\
2
\end{array}\right) a^{n-2} b^{2}+\cdots+\left(\begin{array}{l}
n \\
n
\end{array}\right) a^{0} b^{n},
$$

sendo $\left(\begin{array}{l}n \\ k\end{array}\right)=\frac{n !}{k !(n-k) !}$. Então, para $a=1$ e $b=\frac{1}{n}$, temos:

$$
\begin{aligned}
T_{n} & =\left(1+\frac{1}{n}\right)^{n} \\
& =\left(\begin{array}{l}
n \\
0
\end{array}\right) 1^{n}\left(\frac{1}{n}\right)^{0}+\left(\begin{array}{l}
n \\
1
\end{array}\right) 1^{n-1}\left(\frac{1}{n}\right)^{1}+\left(\begin{array}{l}
n \\
2
\end{array}\right) 1^{n-2}\left(\frac{1}{n}\right)^{2}+\cdots+\left(\begin{array}{l}
n \\
n
\end{array}\right) 1^{0}\left(\frac{1}{n}\right)^{n} \\
& =1+n \cdot \frac{1}{n}+\frac{n(n-1)}{2 !} \cdot \frac{1}{n^{2}}+\cdots+\frac{n(n-1)(n-2) \cdots 1}{n !} \cdot \frac{1}{n^{n}} \\
& =1+1+\left(1-\frac{1}{n}\right) \cdot \frac{1}{2 !}+\cdots+\left(1-\frac{1}{n}\right) \cdot\left(1-\frac{2}{n}\right) \cdots\left(1-\frac{n-1}{n}\right) \cdot \frac{1}{n !} .
\end{aligned}
$$

Observe que as expressões dentro de cada parênteses (na última linha acima) são menores do que 1; assim $T_{n} \leq S_{n}$. Então, podemos concluir que $T_{n}<3$ qualquer que seja $n$. Afirmamos que a sequência $\left(T_{n}\right)$ é crescente. De fato,

$$
T_{n}=1+1+\left(1-\frac{1}{n}\right) \cdot \frac{1}{2 !}+\cdots+\left(1-\frac{1}{n}\right) \cdot\left(1-\frac{2}{n}\right) \cdots\left(1-\frac{n-1}{n}\right) \cdot \frac{1}{n !}
$$

e

$$
\begin{aligned}
T_{n+1} & =1+1+\left(1-\frac{1}{n+1}\right) \cdot \frac{1}{2 !}+\left(1-\frac{1}{n+1}\right) \cdot\left(1-\frac{2}{n+1}\right) \cdot \frac{1}{3 !}+\cdots \\
& +\left(1-\frac{1}{n+1}\right) \cdot\left(1-\frac{2}{n+1}\right) \cdots\left(1-\frac{n-1}{n+1}\right) \cdot \frac{1}{n !} \\
& +\left(1-\frac{1}{n+1}\right) \cdot\left(1-\frac{2}{n+1}\right) \cdots\left(1-\frac{n-1}{n+1}\right) \cdot\left(1-\frac{n}{n+1}\right) \cdot \frac{1}{(n+1) !}
\end{aligned}
$$

Como a última parcela de $\mathrm{T}_{\mathrm{n}+1}$ é maior que zero, temos

$$
\begin{aligned}
T_{n+1} & >1+1+\left(1-\frac{1}{n+1}\right) \cdot \frac{1}{2 !}+\left(1-\frac{1}{n+1}\right) \cdot\left(1-\frac{2}{n+1}\right) \cdot \frac{1}{3 !}+\cdots \\
& +\left(1-\frac{1}{n+1}\right) \cdot\left(1-\frac{2}{n+1}\right) \cdots\left(1-\frac{n-1}{n+1}\right) \cdot \frac{1}{n !}
\end{aligned}
$$


Agora,

$$
\begin{aligned}
\mathrm{n}<\mathrm{n}+1 & \Leftrightarrow \frac{1}{\mathrm{n}}>\frac{1}{\mathrm{n}+1} \Leftrightarrow-\frac{1}{\mathrm{n}}<-\frac{1}{\mathrm{n}+1} \Leftrightarrow-\frac{\mathrm{k}}{\mathrm{n}}<-\frac{\mathrm{k}}{\mathrm{n}+1}, \forall \mathrm{k}>0 \\
& \Leftrightarrow 1-\frac{\mathrm{k}}{\mathrm{n}}<1-\frac{\mathrm{k}}{\mathrm{n}+1}, \forall \mathrm{k}>0 .
\end{aligned}
$$

Em particular, tomando $k=1,2, \cdots, n-1$, temos

$$
\begin{aligned}
T_{n+1} & >1+1+\left(1-\frac{1}{n+1}\right) \cdot \frac{1}{2 !}+\left(1-\frac{1}{n+1}\right) \cdot\left(1-\frac{2}{n+1}\right) \cdot \frac{1}{3 !}+\cdots \\
& +\left(1-\frac{1}{n+1}\right) \cdot\left(1-\frac{2}{n+1}\right) \cdots\left(1-\frac{n-1}{n+1}\right) \cdot \frac{1}{n !} \\
& >1+1+\left(1-\frac{1}{n}\right) \cdot \frac{1}{2 !}+\cdots+\left(1-\frac{n-1}{n}\right) \cdot \frac{1}{n !}=T_{n} .
\end{aligned}
$$

Logo, $T_{n+1}>T_{n}$.

Portanto, como $\left(T_{n}\right)$ é uma seqüência crescente e cada termo $T_{n}$ está entre 2 e 3 , deve existir $T \in \mathbb{R}$, com $2 \leq T \leq 3$, tal que $T_{n}$ se aproxima de $T$ a medida que $n$ aumenta.

Abaixo, segue uma tabela que nos sugere uma aproximação para o valor de $\mathrm{T}$ : 
Tabela 5.2: Aproximação de T

\begin{tabular}{|c|l|c|}
\hline$n$ & $\left(1+\frac{1}{n}\right)^{n}$ & $T_{n}$ \\
\hline 1 & $\left(1+\frac{1}{1}\right)^{1}$ & 2 \\
\hline 2 & $\left(1+\frac{1}{2}\right)^{2}$ & 2,25 \\
\hline 10 & $\left(1+\frac{1}{10}\right)^{10}$ & $\approx 2,59374$ \\
\hline 100 & $\left(1+\frac{1}{100}\right)^{100}$ & $\approx 2,70481$ \\
\hline 1.000 & $\left(1+\frac{1}{1.000}\right)^{1.000}$ & $\approx 2,71692$ \\
\hline 10.000 & $\left(1+\frac{1}{10.000}\right)^{10.000}$ & $\approx 2,71814$ \\
\hline 100.000 & $\left(1+\frac{1}{100.000}\right)^{100.000}$ & $\approx 2,71826$ \\
\hline 1.000 .000 & $\left(1+\frac{1}{1.000 .000}\right)^{1.000 .000}$ & $\approx 2,71828$ \\
\hline 10.000 .000 & $\left(1+\frac{1}{10.000 .000}\right)^{10.000 .000}$ & $\approx 2,71828$ \\
\hline
\end{tabular}

Pela tabela 2 podemos indicar que $\mathrm{T}$ é aproximadamente 2, 718 .

Vamos mostrar que de fato $S=T$. Para isso, vamos mostrar que $S \geq T$ e $S \leq T$.

Para mostrar que $S \geq T$ é simples, pois como $T_{n} \leq S_{n} \leq S$ temos $T \leq S$.

Vamos mostrar, agora, que $S \leq T$. Fixe $m$ e seja $n$ tal que $m<n$. Denotemos por $R_{m, n}$ os primeiros $m+1$ termos de $T_{n}$, isto é,

$$
\mathrm{R}_{\mathrm{m}, \mathrm{n}}=1+1+\left(1-\frac{1}{\mathrm{n}}\right) \cdot \frac{1}{2 !}+\cdots+\left(1-\frac{1}{\mathrm{n}}\right) \cdot\left(1-\frac{2}{\mathrm{n}}\right) \cdot \ldots \cdot\left(1-\frac{\mathrm{m}-1}{\mathrm{n}}\right) \cdot \frac{1}{\mathrm{m!}} .
$$

Note que, como $m<n$ e todos os termos de $\left(T_{n}\right)$ são positivos, $R_{m, n}<T_{n}$. Note também que quando $n$ aumenta $R_{m, n}$ fica perto de $S_{m}$, pois quando $n$ aumenta $1-\frac{1}{n}, 1-\frac{2}{n}, \cdots$, $1-\frac{m-1}{n}$ ficam perto de 1 .

Agora, como $R_{m, n}<T_{n} \leq T$, temos $S_{m} \leq T$ qualquer que seja $m$.

Logo,

$$
\mathrm{S}=\sum_{\mathrm{k}=0}^{\infty} \frac{1}{\mathrm{k} !} \leq \mathrm{T} .
$$


Portanto, $\mathrm{S}=\mathrm{T}$.

Definição 5.3. Definimos $e=\sum_{n=0}^{\infty} \frac{1}{n !}$. Também, $e=\lim _{n \rightarrow \infty}\left(1+\frac{1}{n}\right)^{n}$ e lê-se "limite de $\left(1+\frac{1}{n}\right)^{n}$ quando $n$ tende ao infinito é igual a $e^{\prime \prime}$.

Finalmente, estamos prontos para responder nossa a pergunta: a medida que $\mathrm{n}$ aumenta, qual é o valor de $\left(1+\frac{15}{100 n}\right)^{n}$ ?

Resposta: $e^{\frac{15}{100}}$.

Portanto, um capital de $\mathrm{R} \$ 100,00$ aplicado a uma taxa de $15 \%$ ao longo de um ano deveria ser

$$
\mathrm{R} \$ 100 e^{\frac{15}{100}} \approx \mathrm{R} \$ 116,10
$$

ao final da aplicação. Compare (5.1) e (5.4).

Observação 5.5. A argumentação acima pode ser estendida a qualquer capital $\mathrm{C}_{0}$ aplicado a uma taxa de $\mathrm{t} \%(\mathrm{t} \in \mathbb{Q})$. Ao final de um ano o montante deveria ser

$$
C_{0} e^{\frac{t}{100}}
$$

\section{Verifique!!!}

Observação 5.7. Nossa argumentação mostra que a uma taxa de $15 \%$ tem-se diferença ao calcularmos os juros por (5.1) ou por (5.6). De fato, usando (5.6) podemos verificar que para $t$ pequeno tanto faz aplicar (5.1) ou (5.6), pois vamos obter o mesmo montante final. Porém, para t grande (5.6) fornece um montante maior. Faça simulações!!! Divirta-se!!!

$\mathrm{Na}$ tabela abaixo constam simulações aplicando as fórmulas (5.1) ou (5.6) (adotamos $e=2,718)$. 
Tabela 5.3: Simulações

\begin{tabular}{|c|c|c|c|}
\hline Capital $\mathrm{C}_{0}$ & $\mathrm{t}$ & $C_{0}+\frac{t}{100} C_{0}$ & $\mathrm{C}_{0} \mathrm{e}^{\frac{\mathrm{t}}{100}} \approx \mathrm{C}_{0}(1,01)^{\mathrm{t}}$ \\
\hline 100 & 0,1 & 100,10 & 100,10 \\
\hline 100 & 0,5 & 100,50 & 100,50 \\
\hline 100 & 0,8 & 100,80 & 100,80 \\
\hline 100 & 1 & 101,00 & 101,00 \\
\hline 100 & 2 & 102,00 & 102,01 \\
\hline 100 & 3 & 103,00 & 103,03 \\
\hline 100 & 5 & 105,00 & 105,10 \\
\hline 100 & 10 & 110,00 & 110,46 \\
\hline 100 & 12 & 112,00 & 112,68 \\
\hline 100 & 15 & 115,00 & 116,10 \\
\hline 100 & 20 & 120,00 & 122,02 \\
\hline 100 & 30 & 130,00 & 134,78 \\
\hline 100 & 40 & 140,00 & 148,89 \\
\hline 100 & 50 & 150,00 & 164,46 \\
\hline
\end{tabular}

Observação 5.8. Pelas tabelas 1 e 2 somos levados a concluir que $2<e<3$. Vamos verificar que de fato isto ocorre. Evidentemente, $2<\sum_{n=0}^{\infty} \frac{1}{n !}$. Além disso,

$$
\sum_{n=0}^{\infty} \frac{1}{n !}=1+1+\frac{1}{2}+\frac{1}{6}+\sum_{n=4}^{\infty} \frac{1}{n !}
$$


e, como $\mathrm{n} !=1.2 .3 \cdots \mathrm{n}>1.2 .2 \cdots 2=2^{\mathrm{n}-1}$ para $\mathrm{n} \geq 3$, temos

$$
\sum_{n=0}^{\infty} \frac{1}{n !} \leq 1+1+\frac{1}{2}+\frac{1}{6}+\sum_{n=4}^{\infty} \frac{1}{2^{n-1}} .
$$

Agora, aplicando a fórmula para calcular a soma de uma PG infinita, temos

$$
\sum_{n=4}^{\infty} \frac{1}{2^{n-1}}=\frac{\frac{1}{2^{3}}}{1-\frac{1}{2}}=\frac{1}{4}
$$

Logo,

$$
\sum_{n=0}^{\infty} \frac{1}{n !} \leq 1+1+\frac{1}{2}+\frac{1}{6}+\frac{1}{4}<1+1+\frac{1}{2}+\frac{1}{4}+\frac{1}{4}=3
$$

Portanto, $2<\mathrm{e}<3$.

\subsection{A irracionalidade de $e$}

Nesta seção apresentaremos uma demonstração detalhada da irracionalidade de e, baseada naquela apresentada em [11].

Suponha, por absurdo, que e é um número racional, ou seja, $e=\frac{p}{q}$, com $p$ e q naturais. Podemos supor $\operatorname{mdc}(p, q)=1$. Como $2<e<3$ temos que $e \notin \mathbb{N}$; consequentemente, $q \geq 2$.

Sabemos que

$$
e=\sum_{n=0}^{\infty} \frac{1}{n !}=\frac{1}{0 !}+\frac{1}{1 !}+\frac{1}{2 !}+\frac{1}{3 !}+\cdots
$$

Assim,

$$
\frac{p}{q}=e=1+1+\frac{1}{2 !}+\frac{1}{3 !}+\cdots+\frac{1}{(q-1) !}+\frac{1}{q !}+\frac{1}{(q+1) !}+\cdots+\frac{1}{n !}+\cdots .
$$

Multiplicando a igualdade acima por q!, obtemos

$$
\begin{aligned}
\frac{\mathrm{p}}{\mathrm{q}} \cdot \mathrm{q} ! & =1 \cdot \mathrm{q} !+1 \cdot \mathrm{q} !+\frac{1}{2 !} \cdot \mathrm{q} !+\frac{1}{3 !} \cdot \mathrm{q} !+\cdots+\frac{1}{(\mathrm{q}-1) !} \cdot \mathrm{q} !+\frac{1}{\mathrm{q} !} \cdot \mathrm{q} !+\frac{1}{(\mathrm{q}+1) !} \cdot \mathrm{q} ! \\
& +\cdots+\frac{1}{\mathrm{n} !} \cdot \mathrm{q} !+\cdots
\end{aligned}
$$

Daí,

$$
\begin{aligned}
\frac{p}{q} \cdot q \cdot(q-1) ! & =q !+q !+\frac{1}{2 !} \cdot 1 \cdot 2 \cdot 3 \cdot \ldots \cdot q+\frac{1}{3 !} \cdot 1 \cdot 2 \cdot 3 \cdot \ldots \cdot q+\ldots \\
& +\frac{1}{(q-1) !} \cdot q \cdot(q-1) !+\frac{1}{q !} \cdot q !+\frac{1}{(q+1) \cdot q !} \cdot q !+\cdots+\frac{1}{n !} \cdot q !+\ldots
\end{aligned}
$$


e, consequentemente,

$$
\begin{aligned}
p \cdot(q-1) ! & =q !+q !+3 \cdot 4 \cdot \ldots \cdot q+4 \cdot 5 \cdot \ldots \cdot q+\ldots+q+1+\frac{1}{(q+1)} \\
& +\frac{1}{(q+2)(q+1)}+\cdots+\frac{1}{n !} \cdot q !+\ldots
\end{aligned}
$$

Logo,

$$
\begin{aligned}
\mathrm{p} \cdot(\mathrm{q}-1) !-(\mathrm{q} !+\mathrm{q} !+3 \cdot 4 & \cdot \ldots \cdot \mathrm{q}+4 \cdot 5 \cdot \ldots \cdot \mathrm{q}+\ldots+\mathrm{q}+1) \\
& =\frac{1}{(\mathrm{q}+1)}+\frac{1}{(\mathrm{q}+2)(\mathrm{q}+1)}+\ldots+\frac{1}{\mathrm{n} !} \cdot \mathrm{q} !+\ldots
\end{aligned}
$$

Observe que no lado esquerdo da igualdade (5.9) as parcelas são números inteiros, enquanto que no lado direito as parcelas são frações; além disso, como em (5.9) tem-se $q \geq 2$. Daí, em (5.9) temos

$$
\begin{aligned}
\frac{1}{q+1} & \leq \frac{1}{3} \\
\frac{1}{(q+2)(q+1)} & \leq \frac{1}{3} \cdot \frac{1}{3}=\frac{1}{3^{2}} \\
\frac{1}{n !} \cdot q !=\frac{1}{(q+1) \cdot \ldots \cdot(q+(n-q))} & \leq \frac{1}{3} \cdot \ldots \cdot \frac{1}{3}=\frac{1}{3^{n-q}} .
\end{aligned}
$$

Logo, em (5.9), temos

$$
\frac{1}{q+1}+\frac{1}{(q+2)(q+1)}+\ldots+\frac{1}{n !} \cdot q !+\ldots \leq \frac{1}{3}+\frac{1}{3^{2}}+\ldots+\frac{1}{3^{n-q}}+\ldots
$$

Observe que

$$
\frac{1}{3}+\frac{1}{3^{2}}+\ldots+\frac{1}{3^{n-q}}+\ldots
$$

é uma Progressão Geométrica, sendo que o primeiro termo é $\frac{1}{3}$ e a razão também é $\frac{1}{3}$; logo,

$$
\frac{1}{3}+\frac{1}{3^{2}}+\cdots+\frac{1}{3^{n-q}}+\cdots=\frac{\frac{1}{3}}{1-\frac{1}{3}}=\frac{\frac{1}{3}}{\frac{2}{3}}=\frac{1}{2}
$$

Portanto, segue de (5.9), (5.10) e (5.11) que

$$
p \cdot(q-1) !-(q !+q !+3 \cdot 4 \cdot \ldots \cdot q+4 \cdot 5 \cdot \ldots \cdot q+\ldots+q+1) \leq \frac{1}{2}
$$

Absurdo, pois não existe número inteiro entre 0 e $\frac{1}{2}$. O absurdo ocorreu ao supor que e é número racional; portanto, e é um número irracional. 



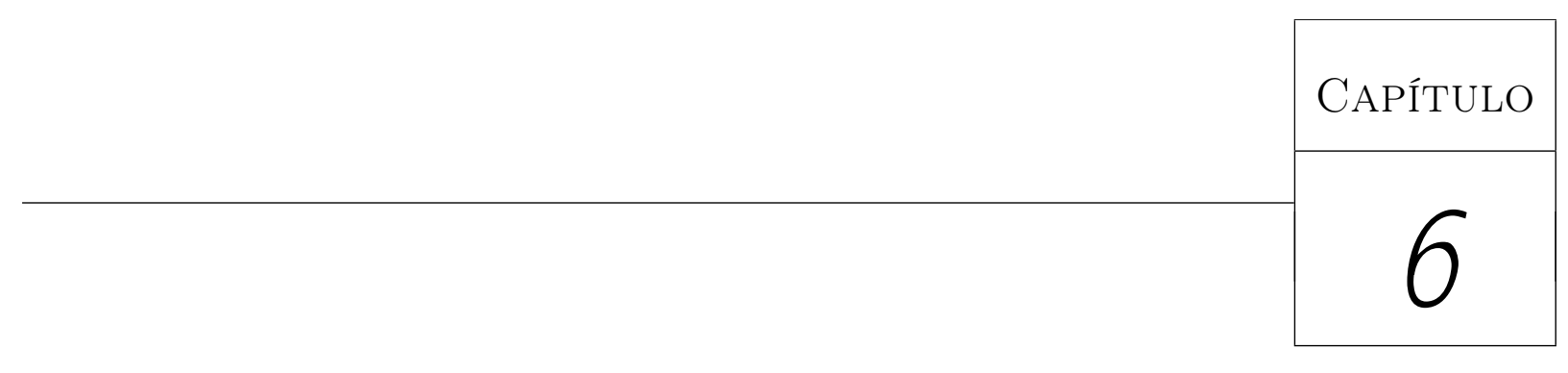

\section{Considerações finais}

A problemática existente na definição do conjunto dos números irracionais no processo de ensino-aprendizagem na educação básica nortearam os objetivos deste trabalho.

Nosso objetivo inicial era estudar as propriedades dos números reais e apresentar uma demonstração da irracionalidade de $\pi$.

No decorrer da pesquisa nos pareceu atraente a ideia de apresentarmos, também, a demonstração da irracionalidade de outro número, a saber, o número e. Este acabou ganhando uma importância que não havíamos previsto inicialmente. De fato, consideramos ser possível apresentar aos alunos do ensino médio tal demonstração. Diante disso, fizemos um estudo das origens, definição e prova da irracionalidade do e, bem como, elaboramos um texto que poderá servir de subsídio para desenvolver o tema em sala de aula.

Através desta dissertação, esperamos levar até o professor de matemática um texto simples, compreensível e que traga segurança ao professor ao abordar o tema números irracionais no ensino fundamental e médio, bem como, ao apresentar o número $\pi$ e 0 número e como exemplos de números irracionais.

Todo o estudo feito para escrever esta dissertação serviu para ampliar minha formação docente, aprofundando meus conhecimentos a respeito da matemática e contribuindo para meu aprimoramento profissional. Através do estudo dos textos indicados nas referências bibliográficas e, principalmente, através das reuniões com o meu orientador, pude contar com uma sólida base teórica que permitiu fundamentar criticamente meus conhecimentos e minha atuação profissional. Acredito estar mais preparada para cumprir meu papel profissional com muito mais segurança. 



\section{Referências Bibliográficas}

[1] Ávila, G., Análise para a Licenciatura, 3a edição, São Paulo: Blucher, 2006.

[2] Barreto F, B., Matemática aula por aula, 1. ${ }^{a}$ edição, São Paulo:FTD, 2003.

[3] Bianchini, E. Matemática, $1^{\text {a }}$ edição, São Paulo: Moderna, 2004.

[4] Dante, L.R., Matemática: Livro do Professor, 1.a edição São Paulo: Ática, 2004.

[5] Evaristo, J. e Perdigão, E., Introdução a Álgebra Abstrata, Alagoas: Edufal, 2002.

[6] Figueiredo, D.G., Números Irracionais e transcendentes, Sociedade Brasileira de Matemática, Brasília, 1985.

[7] Guidorizzi, H.L., Um curso de cálculo, Volume 1, 5 ${ }^{\mathrm{a}}$ Edição, Rio de Janeiro: LTC, 2001.

[8] Giovani, J.R., Matemática completa, 2. ${ }^{a}$ edição, São Paulo: FTD, 2005.

[9] Hefez, A., Elementos de aritmética, $2^{\mathrm{a}}$ Edição, Rio de Janeiro: SBM, 2011.

[10] Lima, E.L., Curso de Análise, vol. 1, Projeto Euclides, IMPA, Rio de Janeiro RJ, 1976.

[11] Maor, E., e: A História de um Número, $5^{\mathrm{a}}$ edição, Trad. Jorge Calife, Rio de Janeiro: Editora Record, 2008.

[12] Niven, I., A simple proof that is irrational, Bulletin of the American Mathematical Society, 53(6), 509, 1947.

[13] Niven, I., Números Racionais e Irracionais, Second Edition (1984), Sociedade Brasileira de Matemática, Rio de Janeiro-RJ.

[14] Rudin, W., Principles of Mathematical Analysis, McGraw-Hill, 1976.

[15] Smole, K.C.S. Matemática - ensino médio - 1ª edição, São Paulo: Saraiva, 2005. 